\title{
The Zeta And Related Functions: Recent DEVELOPMENTS
}

\author{
H. M. SRIVASTAVA $A^{1,2, *}$ \\ ${ }^{1}$ Department of Mathematics and Statistics, University of Victoria, \\ Victoria, British Columbia V8W 3R4, Canada \\ ${ }^{2}$ Department of Medical Research, China Medical University Hospital, China Medical University, \\ Taichung 40402, Taiwan, Republic of China \\ *Corresponding Author: H. M. SRIVASTAVA (Email: harimsri@math.uvic.ca) \\ (Received: 21-Jan-2019; accepted: 22-Feb-2019; published: 31-Mar-2019) \\ DOI: http://dx.doi.org/10.25073/jaec.201931.229
}

\begin{abstract}
The main object of this surveycum-expository article is to present an overview of some recent developments involving the Riemann Zeta function $\zeta(s)$, the Hurwitz (or generalized) Zeta function $\zeta(s, a)$, and the HurwitzLerch Zeta function $\Phi(z, s, a)$, which have their roots in the works of the great eighteenth-century Swiss mathematician, Leonhard Euler (17071783) and the Russian mathematician, Christian Goldbach (1690-1764). We aim at considering the problems associated with the evaluations and representations of $\zeta(s)$ when $s \in$ $\mathbb{N} \backslash\{1\}, \mathbb{N}$ being the set of natural numbers, with emphasis upon several interesting classes of rapidly convergent series representations for $\zeta(2 n+1)(n \in \mathbb{N})$. Symbolic and numerical computations using Mathematica (Version 4.0) for Linux will also be provided for supporting their computational usefulness.
\end{abstract}

\section{Keywords}

Riemann Zeta Function; Hurwitz (or generalized) Zeta Function; Hurwitz-Lerch Zeta Function; Associated Series and Integrals; Analytic Number Theory; Series Representations; Harmonic Numbers; Bernoulli Numbers and Bernoulli Polynomials; Generating Functions; Euler Numbers and Euler Polynomials; In- ductive Argument; Symbolic and Numerical Computations; Euler Sums.

\section{Introduction, Definitions and Preliminaries}

Throughout this lecture, we use the following standard notations:

$\mathbb{N}:=\{1,2,3, \cdots\}, \quad \mathbb{N}_{0}:=\{0,1,2, \cdots\}=\mathbb{N} \cup\{0\}$

and

$$
\mathbb{Z}^{-}:=\{-1,-2,-3, \cdots\}=\mathbb{Z}_{0}^{-} \backslash\{0\} .
$$

Also, as usual, $\mathbb{Z}$ denotes the set of integers, $\mathbb{R}$ denotes the set of real numbers, $\mathbb{R}^{+}$denotes the set of positive numbers and $\mathbb{C}$ denotes the set of complex numbers.

Some rather important and potentially useful functions in Analytic Number Theory include (for example) the Riemann Zeta function $\zeta(s)$ and the Hurwitz (or generalized) Zeta function 
$\zeta(s, a)$, which are defined (for $\Re(s)>1$ ) by

$$
\zeta(s):=\left\{\begin{array}{l}
\sum_{n=1}^{\infty} \frac{1}{n^{s}}=\frac{1}{1-2^{-s}} \sum_{n=1}^{\infty} \frac{1}{(2 n-1)^{s}} \\
(\Re(s)>1) \\
\frac{1}{1-2^{1-s}} \sum_{n=1}^{\infty} \frac{(-1)^{n-1}}{n^{s}} \\
(\Re(s)>0 ; s \neq 1)
\end{array}\right.
$$

and

$\zeta(s, a):=\sum_{n=0}^{\infty} \frac{1}{(n+a)^{s}},\left(\Re(s)>1 ; a \in \mathbb{C} \backslash \mathbb{Z}_{0}^{-}\right)$,

and (for $\Re(s) \leqq 1 ; s \neq 1$ ) by their meromorphic continuations (see, for details, the excellent works by Titchmarsh [72] and Apostol [4] as well as the monumental treatise by Whittaker and Watson [75]; see also [1, Chapter 23] and [57, Chapter 2]), so that (obviously)

$$
\zeta(s, 1)=\zeta(s)=\left(2^{s}-1\right)^{-1} \zeta\left(s, \frac{1}{2}\right)
$$

and

$$
\zeta(s, 2)=\zeta(s)-1
$$

with, of course,

$$
\zeta(0, a)=\frac{1}{2}-a, \quad \zeta(0)=-\frac{1}{2}
$$

and

$$
\zeta(-2 n)=0 \quad(n \in \mathbb{N}) .
$$

More generally, we have the following relationships:

$$
\zeta(s)=\frac{1}{m^{s}-1} \sum_{j=1}^{m-1} \zeta\left(s, \frac{j}{m}\right),(m \in \mathbb{N} \backslash\{1\})
$$

and

$$
\zeta(s, m a)=\frac{1}{m^{s}} \sum_{j=0}^{m-1} \zeta\left(s, a+\frac{j}{m}\right),(m \in \mathbb{N}) .
$$

Generally speaking, Mathematics appeals to the intellect. In addition, however, great mathematics possesses a kind of perceptual quality which endows it with a beauty comparable to that of great art or great music. Much of the work of the 18th century Swiss mathematician, Leonhard Euler (1707-1783), belongs in this category. Euler's work on $\zeta(s)$ began around 1730 with approximations to the value of $\zeta(2)$, continued with the evaluation of $\zeta(2 n) \quad(n \in \mathbb{N})$, and resulted around 1749 in the discovery of the celebrated functional equation for $\zeta(s)$ almost 110 years before the remarkably influential German mathematician, Georg Friedrich Bernhard Riemann (1826-1866).

A classical about three-century-old theorem of Christian Goldbach (1690-1764) was stated in a letter dated 1729 from Goldbach to Daniel Bernoulli (1700-1782). Goldbach's Theorem (or, equivalently, the Goldbach-Euler Theorem) was revived and revisited recently as the following problem in many publications such as (for example)

[a] J. D. Shallit and K. Zikan, A theorem of Goldbach, Amer. Math. Monthly 93 (1986), 402-403

and

[b] J. K. Haughlund, D. Tjaden and J. Groeneveld, Problem 38, Nieuw Arch. Wisk. (Ser. 5) 4 (2003), 95-96.

$$
\sum_{\omega \in \mathcal{S}} \frac{1}{\omega-1}=1
$$

where $\mathcal{S}$ denotes the set of all nontrivial integer $k$ th powers, that is,

$$
\begin{aligned}
\mathcal{S} & :=\left\{n^{k}: n, k \in \mathbb{N} \backslash\{1\}\right\} \\
& =\{4,8,9,16,25,27,32,36, \cdots\} .
\end{aligned}
$$

\section{What does Goldbach's Theorem (5) have to do with the Riemann Zeta function $\zeta(s)$ defined by (1)?}

In order to answer this question, let $\mathcal{T}$ denote the set of all positive integers that are not in $\mathcal{S}$ other than 1 , that is,

$$
\mathcal{T}:=\{\tau: \tau \notin \mathcal{S} \quad \text { and } \quad \tau \in \mathbb{N} \backslash\{1\}\} .
$$


We then find that

$$
\begin{aligned}
\sum_{\omega \in \mathcal{S}} \frac{1}{\omega-1} & =\sum_{k=2}^{\infty} \sum_{\tau \in \mathcal{T}}\left(\tau^{k}-1\right)^{-1} \\
& =\sum_{k=2}^{\infty} \sum_{\tau \in \mathcal{T}} \sum_{j=1}^{\infty} \tau^{-j k} \\
& =\sum_{k=2}^{\infty} \sum_{j=1}^{\infty} \sum_{\tau \in \mathcal{T}}\left(\tau^{j}\right)^{-k} \\
& =\sum_{k=2}^{\infty} \sum_{n=2}^{\infty} \frac{1}{n^{k}} \\
& =\sum_{k=2}^{\infty}(\zeta(k)-1),
\end{aligned}
$$

since it is easily seen that

$$
\begin{aligned}
& \sum_{k=2}^{\infty} \sum_{j=1}^{\infty} \sum_{\tau \in \mathcal{T}}\left(\tau^{j}\right)^{-k} \\
& =\sum_{k=2}^{\infty} \sum_{j=1}^{\infty}\left[\begin{array}{l}
\left(2^{j}\right)^{-k}+\left(3^{j}\right)^{-k}+\left(5^{j}\right)^{-k} \\
+\left(6^{j}\right)^{-k}+\left(7^{j}\right)^{-k}+\left(10^{j}\right)^{-k} \\
+\left(11^{j}\right)^{-k}+\left(12^{j}\right)^{-k}+\cdots
\end{array}\right] \\
& =\sum_{k=2}^{\infty}\left[\begin{array}{l}
\left(2^{-k}+3^{-k}+5^{-k}+6^{-k}+7^{-k}+\cdots\right) \\
+\left(4^{-k}+9^{-k}+25^{-k}+36^{-k}+\cdots\right) \\
+\left(8^{-k}+27^{-k}+125^{-k}+\cdots\right)+\cdots
\end{array}\right] \\
& =\sum_{k=2}^{\infty} \sum_{n=2}^{\infty} n^{-k}=\sum_{k=2}^{\infty}(\zeta(k)-1) .
\end{aligned}
$$

Thus, in terms of the Riemann Zeta function $\zeta(s)$ defined by (1), Goldbach's Theorem (5) is easily seen to assume the following elegant form:

$$
\sum_{\omega \in \mathcal{S}} \frac{1}{\omega-1}=\sum_{k=2}^{\infty}(\zeta(k)-1)=1 .
$$

Since $\zeta(s)$ is a decreasing function of its argument $s$ for $s \geqq 2$, we have

$$
1<\zeta(n) \leqq \zeta(2)=\frac{\pi^{2}}{6}<2,
$$

the above alternative form (6) of Goldbach's Theorem (5) can also be rewritten as follows:

$$
\sum_{k=2}^{\infty} \mathfrak{f}(\zeta(k))=1
$$

where

$$
\mathfrak{f}(x):=x-[x]=\text { The fractional part of } x \in \mathbb{R} .
$$

As a matter of fact, it is fairly straightforward to show also that

$$
\begin{aligned}
& \sum_{k=2}^{\infty}(-1)^{k} \mathfrak{f}(\zeta(k))=\frac{1}{2}, \\
& \sum_{k=1}^{\infty} \mathfrak{f}(\zeta(2 k))=\frac{3}{4}, \\
& \sum_{k=1}^{\infty} \mathfrak{f}(\zeta(2 k+1))=\frac{1}{4}, \\
& \sum_{k=1}^{\infty} \mathfrak{f}(\zeta(4 k))=\frac{1}{8}(7-2 \operatorname{coth} \pi),
\end{aligned}
$$

and so on.

Several extensions and generalizations of Goldbach's Theorem (5) have been investigated. For example, we recall the following generalizations given in

[c] J. Choi and H. M. Srivastava, Series involving the Zeta functions and a family of generalized Goldbach-Euler series, Amer. Math. Monthly 121 (2014), 229-236.

$$
\begin{gathered}
\sum_{\omega \in \mathcal{S}_{p, 0}} \frac{1}{\omega-1}=\frac{1}{p}\left[\psi(1)-\psi\left(1-\frac{1}{p}\right)\right], \\
(p \in \mathbb{N} \backslash\{1\})
\end{gathered}
$$

and

$$
\sum_{\omega \in \mathcal{S}_{p, 1}} \frac{1}{\omega-1}=1+\frac{1}{p}\left[\psi\left(\frac{1}{p}\right)-\psi(1)\right],(p \in \mathbb{N}),
$$

where the set $\mathcal{S}_{p, 0}$ is defined (for fixed $p \in \mathbb{N} \backslash$ $\{1\}$ ) by

$$
\mathcal{S}_{p, 0}:=\left\{(p n)^{k}: n \in \mathbb{N} \text { and } k \in \mathbb{N} \backslash\{1\}\right\}
$$

and the set $\mathcal{S}_{p, 1}$ is defined (for fixed $p \in \mathbb{N}$ ) by

$$
\mathcal{S}_{p, 1}:=\left\{(p n+1)^{k}: n \in \mathbb{N} \text { and } k \in \mathbb{N} \backslash\{1\}\right\},
$$

and the Psi (or Digamma) function $\psi(z)$ is defined (as usual) by

$$
\psi(z):=\frac{d}{d z}\{\log \Gamma(z)\}=\frac{\Gamma^{\prime}(z)}{\Gamma(z)}
$$


or

$$
\log \Gamma(z)=\int_{1}^{z} \psi(t) \mathrm{d} t
$$

In fact, in terms of the Riemann zeta function $\zeta(s)$ and Hurwitz (or generalized) zeta function $\zeta(s, a)$, we have

$$
\begin{aligned}
\sum_{\omega \in \mathcal{S}_{p, 0}} \frac{1}{\omega-1} & =\sum_{k=2}^{\infty} \frac{\zeta(k)}{p^{k}} \\
& =\frac{1}{p}\left[\psi(1)-\psi\left(1-\frac{1}{p}\right)\right],
\end{aligned}
$$

$(p \in \mathbb{N} \backslash\{1\})$ and

$$
\begin{aligned}
\sum_{\omega \in \mathcal{S}_{p, 1}} \frac{1}{\omega-1} & =\sum_{k=2}^{\infty} \frac{1}{p^{k}} \zeta\left(k, 1+\frac{1}{p}\right) \\
& =1+\frac{1}{p}\left[\psi\left(\frac{1}{p}\right)-\psi(1)\right]
\end{aligned}
$$

$(p \in \mathbb{N})$ or, equivalently,

$$
\begin{aligned}
& \sum_{k=2}^{\infty} \sum_{n=1}^{\infty} \frac{1}{(p n)^{k}-1}=\sum_{k=2}^{\infty} \sum_{j=1}^{\infty} \frac{1}{p^{k j}} \zeta(k j) \\
& =\sum_{\omega \in \mathcal{S}_{p, 0}} \frac{1}{\omega-1}+\sum_{k=2}^{\infty} \sum_{j=2}^{\infty} \frac{1}{p^{k j}} \zeta(k j)
\end{aligned}
$$

$(p \in \mathbb{N} \backslash\{1\})$, and

$$
\begin{aligned}
& \sum_{k=2}^{\infty} \sum_{n=1}^{\infty} \frac{1}{(p n+1)^{k}-1} \\
& =\sum_{k=2}^{\infty} \sum_{j=1}^{\infty} \frac{1}{p^{k j}} \zeta\left(k j, 1+\frac{1}{p}\right) \\
& =\sum_{\omega \in \mathcal{S}_{p, 1}} \frac{1}{\omega-1}+\sum_{k=2}^{\infty} \sum_{j=2}^{\infty} \frac{1}{p^{k j}} \zeta\left(k j, 1+\frac{1}{p}\right),
\end{aligned}
$$

$(p \in \mathbb{N})$, respectively. This last pair of the Goldbach-Euler type sums poses a natural question as the following open problem.

Open Problem. For each of the following double sums:

$$
\begin{aligned}
& \sum_{k=2}^{\infty} \sum_{j=1}^{\infty} \frac{1}{p^{k j}} \zeta(k j) \\
& \sum_{k=2}^{\infty} \sum_{j=1}^{\infty} \frac{1}{p^{k j}} \zeta\left(k j, 1+\frac{1}{p}\right)
\end{aligned}
$$

find a closed-form evaluation or expression.

The name of Christian Goldbach (1690-1764) is usually associated with a relatively more popular conjecture first proposed in a letter dated 1742 from Goldbach to Euler (known as Goldbach's Conjecture), that is,

Every positive even integer greater than 2 is the sum of two prime numbers:

$$
\begin{aligned}
& 4=2+2=1+3 \\
& 6=3+3=1+5 ; \\
& 8=1+7=3+5 ; \quad \text { et cetera. }
\end{aligned}
$$

Just as the celebrated Riemann Hypothesis dated 1859 that all nontrivial zeros of $\zeta(s)$ lie on the critical line:

$$
\Re(s)=\frac{1}{2},
$$

Goldbach's conjecture has not been proven as yet. Interestingly, not too long ago in the year 2001, on the occasion of the publication of the following ("very funny, tender, charming, and irresistible") novel:

Uncle Pedros and Goldbach's Conjecture: A Novel of Mathematical Obsession (by Apostolos Doxiadis), Faber and Faber, London, 2001.

the British publisher (Faber and Faber) had offered a reward of one million U.K. Pounds to anyone who can prove Goldbach's Conjecture.

Another result that has attracted fascinatingly and tantalizingly large number of seemingly independent solutions is the so-called Basler Problem or Basel Problem:

$$
\zeta(2):=\sum_{k=1}^{\infty} \frac{1}{k^{2}}=\frac{\pi^{2}}{6},
$$

which was used above in (7). It was of vital importance to Leonhard Euler (1707-1783) and the Bernoulli brothers [Jakob Bernoulli (16541705) and Johann Bernoulli (1667-1748)]. Remarkably many (over a couple of dozen) essen- 
tially independent solutions the Basler Problem (8) have appeared in the mathematical literature ever since Euler first solved this problem in the year 1736 .

The city of Basel in Switzerland was one of many free cities in Europe. By the 17th century, it had become an important center of trade and commerce. The University of Basel became a noted institution in Europe, largely through the fame of an extraordinary family, namely, the Bernoullis. This family had come from Antwerp to Basel. The founder of the Bernoulli mathematical dynasty was Nicolaus (Nicholas) Bernoulli who was a painter and Alderman of Basel. He had 3 sons; two of his sons, Jakob [often referred to as James] (1654-1705) and Johann [often referred to as John] (1667-1748), became noted mathematicians. Both were pupils of Gottfried Wilhelm von Leibniz (1646-1716) with whom Johann Bernoulli carried on an extensive correspondence and with whose work both Jacob Bernoulli and Johann Bernoulli became familiar. Jacob Bernoulli was a professor at the University of Basel until his death in 1705. Johann Bernoulli, who had been a professor at the University of Groningen in the (present-day) Netherlands, replaced his brother at the University of Basel. Johann Bernoulli had 3 sons. Two of them, Nicholas II (1695-1726) and Daniel (1700-1782), were mathematicians who befriended Euler. They both went to the Academy in St. Petersburg in 1725 and they both had a high regard for their younger colleague, Euler. After some effort, Daniel wrote to Euler that he had secured for him a stipend in the Academy. The appointment for Euler was actually in the physiology section, but Euler quickly drifted into the mathematics section. He thus left Basel for St. Petersburg in 1727 and remained there until 1741 when he was summoned by Frederick the Great of Prussia to the Berlin Academy. Euler was in Berlin until 1766 when he was summoned back to the Academy in St. Petersburg where he remained until his death in 1783. Euler carried on an extensive correspondence with various mathematicians, especially with Christian Goldbach (1690-1764). He also wrote a series of letters on various subjects in natural philosophy and addressed these letters to a German princess. The quality of all his letters reflects Euler's pleasant personality.

In the above context, one other remarkable classical result involving Riemann's Zeta function $\zeta(s)$ is the following elegant series representation for $\zeta(3)$ :

$$
\zeta(3)=-\frac{4 \pi^{2}}{7} \sum_{k=0}^{\infty} \frac{\zeta(2 k)}{(2 k+1)(2 k+2) \cdot 2^{2 k}},
$$

which was actually contained in Euler's 1772 paper entitled "Exercitationes Analyticae" (cf., e.g., Ayoub [5, pp. 1084-1085]). In fact, This 1772 result of Euler was rediscovered (among others) by Ramaswami [44] (see also a paper by Srivastava [47, p. 7, Equation (35)]) and (more recently) by Ewell [19]. Moreover, just as pointed out by (for example) Chen and Srivastava [7, pp. 180-181], another series representation:

$$
\zeta(3)=\frac{5}{2} \sum_{k=1}^{\infty} \frac{(-1)^{k-1}}{k^{3}\left(\begin{array}{c}
2 k \\
k
\end{array}\right)},
$$

which played a key rôle in the celebrated proof (see, for details, [3]) of the irrationality of $\zeta(3)$ by Roger Apéry (1916-1994), was derived independently by (among others) Hjortnaes [28], Gosper [24], and Apéry [3]. Such elegant expressions as in (10) are known also for $\zeta(2)$ and $\zeta(4):$

$$
\zeta(2)=3 \sum_{k=1}^{\infty} \frac{1}{k^{2}\left(\begin{array}{c}
2 k \\
k
\end{array}\right)}
$$

and

$$
\zeta(4)=\frac{36}{17} \sum_{k=1}^{\infty} \frac{1}{k^{4}\left(\begin{array}{c}
2 k \\
k
\end{array}\right)} .
$$

No such single-term sum expressions are known for $\zeta(n)$ when $n \geqq 5$.

It is easily observed that Euler's series in (9) converges faster than the defining series for $\zeta(3)$, but obviously not as fast as the series in (10). In fact, the order estimates for their general terms are given as follows:

$$
\zeta(3)=-\frac{4 \pi^{2}}{7} \sum_{k=0}^{\infty} \frac{\zeta(2 k)}{(2 k+1)(2 k+2) 2^{2 k}},
$$




$$
\left[O\left(k^{-2} \cdot 2^{-2 k}\right)(k \rightarrow \infty)\right]
$$

and

$$
\begin{aligned}
& \zeta(3)=\frac{5}{2} \sum_{k=1}^{\infty} \frac{(-1)^{k-1}}{k^{3}\left(\begin{array}{c}
2 k \\
k
\end{array}\right)}, \\
& {\left[O\left(k^{-\frac{5}{2}} \cdot 2^{-2 k}\right) \quad(k \rightarrow \infty)\right] .}
\end{aligned}
$$

It is especially remarkable that Euler was already blind when he performed the breathtaking calculations leading to his result (9) rather mentally.

Evaluations of such Zeta values as $\zeta(3), \zeta(5)$, et cetera are known to arise naturally in a wide variety of applications such as those in Elastostatics, Quantum Field Theory, et cetera (see, for example, Tricomi [73], Witten [77], and Nash and O'Connor [39], [40]). On the other hand, in the case of even integer arguments, we already have the following computationally useful relationship:

$$
\zeta(2 n)=(-1)^{n-1} \frac{(2 \pi)^{2 n}}{2 \cdot(2 n) !} B_{2 n}
$$

$\left(n \in \mathbb{N}_{0}:=\mathbb{N} \cup\{0\}\right)$ with the well-tabulated Bernoulli numbers defined by the following generating function:

$$
\frac{z}{e^{z}-1}=\sum_{n=0}^{\infty} B_{n} \frac{z^{n}}{n !} \quad(|z|<2 \pi),
$$

as well as by the familiar recursion formula:

$$
\zeta(2 n)=\left(n+\frac{1}{2}\right)^{-1} \sum_{k=1}^{n-1} \zeta(2 k) \zeta(2 n-2 k),
$$

$(n \in \mathbb{N} \backslash\{1\})$. Our presentation in this lecture consists of two major parts. First of all, motivated essentially by a genuine need (for computational purposes) for expressing $\zeta(2 n+1)$ as a rapidly converging series for all $n \in \mathbb{N}$, we propose to present a rather systematic investigation of the various interesting families of rapidly convergent series representations for the Riemann $\zeta(2 n+1)(n \in \mathbb{N})$. Relevant connections of the results presented here with many other known series representations for $\zeta(2 n+1)(n \in \mathbb{N})$ are also briefly indicated. In fact, for two of the many computationally useful special cases considered here, we observe that $\zeta(3)$ can be represented by means of series which converge much more rapidly than that in Euler's celebrated formula (9) as well as that in the series (10) which was used recently by Apéry [3] in his proof of the irrationality of $\zeta(3)$. Symbolic and numerical computations using Mathematica (Version 4.0) for Linux show, among other things, that only 50 terms of one of these series are capable of producing an accuracy of seven decimal places. In the second part of this lecture, we consider a variety of series and integrals associated with the Hurwitz-Lerch Zeta function $\Phi(z, s, a)$ as well as its various interesting extensions and generalizations (see Section 6).

\section{Series Representations for $\zeta(2 n+1)(n \in \mathbb{N})$}

The following simple consequence of the binomial theorem and the definition (1):

$$
\sum_{k=0}^{\infty} \frac{(s)_{k}}{k !} \zeta(s+k, a) t^{k}=\zeta(s, a-t) \quad(|t|<|a|)
$$

yields, for $a=1$ and $t= \pm 1 / m$, a useful the series identity in the form:

$$
\sum_{k=0}^{\infty} \frac{(s)_{2 k}}{(2 k) !} \frac{\zeta(s+2 k)}{m^{2 k}}
$$

$$
=\left\{\begin{array}{l}
\left(2^{s}-1\right) \zeta(s)-2^{s-1} \\
(m=2) \\
\frac{1}{2}\left[\left(m^{s}-1\right) \zeta(s)-m^{s}-\sum_{j=2}^{m-2} \zeta\left(s, \frac{j}{m}\right)\right] \\
(m \in \mathbb{N} \backslash\{1,2\})
\end{array}\right.
$$

where $(\lambda)_{\nu}$ denotes the general Pochhammer symbol or the shifted factorial, since

$$
(1)_{n}=n ! \quad\left(n \in \mathbb{N}_{0}\right),
$$


which is defined, in terms of the familiar Gamma with, of course,

function, by

$(\lambda)_{\nu}:=\frac{\Gamma(\lambda+\nu)}{\Gamma(\lambda)}=\left\{\begin{array}{l}1, \quad(\nu=0 ; \lambda \in \mathbb{C} \backslash\{0\} \\ \lambda(\lambda+1) \cdots(\lambda+n-1) \\ (\nu=n \in \mathbb{N} ; \lambda \in \mathbb{C}),\end{array}\right.$

$\zeta(0)=-\frac{1}{2} ; \zeta(-2 n)=0(n \in \mathbb{N}) ;$

$\zeta^{\prime}(0)=-\frac{1}{2} \log (2 \pi)$,

and each of the following limit relationships:

$$
\lim _{s \rightarrow-2 n}\left\{\frac{\sin \left(\frac{1}{2} \pi s\right)}{s+2 n}\right\}=(-1)^{n} \frac{\pi}{2} \quad(n \in \mathbb{N})
$$

1 and assumed tacitly that the $\Gamma$-quotient exists (See, for details, [48] and [57]).

and

Making use of the familiar harmonic numbers $H_{n}$ given by

$$
H_{n}:=\sum_{j=1}^{n} \frac{1}{j} \quad(n \in \mathbb{N}),
$$

the following set of series representations for $\zeta(2 n+1) \quad(n \in \mathbb{N})$ were proven by Srivastava [51] by appealing appropriately to the series identity (15) in its special cases when $m=$ $2,3,4$, and 6 , and also to many other properties and characteristics of the Riemann Zeta function such as the familiar functional equation for $\zeta(s)$ (which was discovered by Euler around 1749, that is, almost 110 years before Riemann):

$$
\zeta(s)=2 \cdot(2 \pi)^{s-1} \sin \left(\frac{1}{2} \pi s\right) \Gamma(1-s) \zeta(1-s)
$$

or, equivalently,

$$
\zeta(1-s)=2 \cdot(2 \pi)^{-s} \cos \left(\frac{1}{2} \pi s\right) \Gamma(s) \zeta(s),
$$

the familiar derivative formula:

$$
\begin{aligned}
\zeta^{\prime}(-2 n) & =\lim _{\varepsilon \rightarrow 0}\left\{\frac{\zeta(-2 n+\varepsilon)-\zeta(-2 n)}{\varepsilon}\right\} \\
& =\lim _{\varepsilon \rightarrow 0}\left\{\frac{\zeta(-2 n+\varepsilon)}{\varepsilon}\right\} \\
& =\frac{(-1)^{n}}{2 \cdot(2 \pi)^{2 n}}(2 n) ! \zeta(2 n+1),(n \in \mathbb{N}),
\end{aligned}
$$

so that

$$
\begin{aligned}
\zeta^{\prime}(-2) & =-\frac{\zeta(3)}{4 \pi^{2}}, \zeta^{\prime}(-4)=\frac{3 \zeta(5)}{4 \pi^{4}} \\
\zeta^{\prime}(-6) & =\frac{45 \zeta(7)}{4 \pi^{6}}, \zeta^{\prime}(-8)=\frac{315 \zeta(9)}{4 \pi^{8}}, \cdots
\end{aligned}
$$

$$
\begin{aligned}
& \lim _{s \rightarrow-2 n}\left\{\frac{\zeta(s+2 k)}{s+2 n}\right\} \\
& =\frac{(-1)^{n-k}}{2 \cdot(2 \pi)^{2(n-k)}}(2 n-2 k) ! \zeta(2 n-2 k+1) . \\
& (k=1, \ldots, n-1 ; n \in \mathbb{N} \backslash\{1\})
\end{aligned}
$$

First Series Representation:

$$
\begin{aligned}
& \zeta(2 n+1)=(-1)^{n-1} \frac{(2 \pi)^{2 n}}{2^{2 n+1}-1}\left[\frac{H_{2 n}-\log \pi}{(2 n) !}\right. \\
& \left.+\sum_{k=1}^{n-1} \frac{(-1)^{k}}{(2 n-2 k) !} \frac{\zeta(2 k+1)}{\pi^{2 k}}\right],(n \in \mathbb{N}) \quad(22) \\
& \left.+2 \sum_{k=1}^{\infty} \frac{(2 k-1) !}{(2 n+2 k) !} \frac{\zeta(2 k)}{2^{2 k}}\right]
\end{aligned}
$$

\section{Second Series Representation:}

$$
\begin{aligned}
& \zeta(2 n+1)=(-1)^{n-1} \frac{2 \cdot(2 \pi)^{2 n}}{3^{2 n+1}-1}\left[\frac{H_{2 n}-\log \left(\frac{2}{3} \pi\right)}{(2 n) !}\right. \\
& \left.+2 \sum_{k=1}^{\infty} \frac{(2 k-1) !}{(2 n+2 k) !} \frac{\zeta(2 k)}{3^{2 k}}\right],(n \in \mathbb{N}) \quad(23) \\
& \left.+\sum_{k=1}^{n-1} \frac{(-1)^{k}}{(2 n-2 k) !} \frac{\zeta(2 k+1)}{\left(\frac{2}{3} \pi\right)^{2 k}}\right]
\end{aligned}
$$

\section{Third Series Representation:}

$$
\begin{gathered}
\zeta(2 n+1)=(-1)^{n-1} \frac{2 \cdot(2 \pi)^{2 n}}{2^{4 n+1}+2^{2 n}-1} \\
\cdot\left[\begin{array}{l}
\frac{H_{2 n}-\log \left(\frac{1}{2} \pi\right)}{(2 n) !} \\
+\sum_{k=1}^{n-1} \frac{(-1)^{k}}{(2 n-2 k) !} \\
+2 \sum_{k=1}^{\infty} \frac{(2 k-1) !}{(2 n+2 k) !} \frac{\zeta(2 k)}{\left(\frac{1}{2} \pi\right)^{2 k}}
\end{array}\right],(n \in \mathbb{N})
\end{gathered}
$$




\section{Fourth Series Representation:}

$$
\left.\begin{array}{c}
\zeta(2 n+1)=(-1)^{n-1} \frac{2 \cdot(2 \pi)^{2 n}}{3^{2 n}\left(2^{2 n}+1\right)+2^{2 n}-1} \\
\cdot\left[\begin{array}{l}
\frac{H_{2 n}-\log \left(\frac{1}{3} \pi\right)}{(2 n) !} \\
+\sum_{k=1}^{n-1} \frac{(-1)^{k}}{(2 n-2 k) !} \frac{\zeta(2 k+1)}{\left(\frac{1}{3} \pi\right)^{2 k}} \\
+2 \sum_{k=1}^{\infty} \frac{(2 k-1) !}{(2 n+2 k) !}
\end{array}\right],(n \in \mathbb{N}) \\
6^{2 k}
\end{array}\right]
$$

Here, as well as elsewhere in this presentation, an empty sum is understood (as usual) to be zero.

The first series representation (22) is markedly different from each of the series representations for $\zeta(2 n+1)$, which were given earlier by Zhang and Williams [79, p. 1590, Equation (54)] and (subsequently) by Cvijović and Klinowski [14, p. 1265, Theorem A] (see also [80] and [81]). Since $\zeta(2 k) \rightarrow 1$ as $k \rightarrow \infty$, the general term in the series representation (22) has the following order estimate:

$$
O\left(2^{-2 k} \cdot k^{-2 n-1}\right) \quad(k \rightarrow \infty ; n \in \mathbb{N}),
$$

whereas the general term in each of the aforecited earlier series representations has the order estimate given below:

$$
O\left(2^{-2 k} \cdot k^{-2 n}\right) \quad(k \rightarrow \infty ; n \in \mathbb{N}) .
$$

In case we suitably combine (22) and (24), we readily obtain the following series representation:

$$
\begin{gathered}
\zeta(2 n+1)=(-1)^{n-1} \frac{2 \cdot(2 \pi)^{2 n}}{\left(2^{2 n}-1\right)\left(2^{2 n+1}-1\right)} \\
{\left[\begin{array}{c}
\frac{\log 2}{(2 n) !}+\sum_{k=1}^{n-1} \frac{(-1)^{k}\left(2^{2 k}-1\right)}{(2 n-2 k) !} \frac{\zeta(2 k+1)}{\pi^{2 k}} \\
-2 \sum_{k=1}^{\infty} \frac{(2 k-1) !\left(2^{2 k}-1\right)}{(2 n+2 k) !} \frac{\zeta(2 k)}{2^{4 k}}
\end{array}\right],}
\end{gathered}
$$

where $n \in \mathbb{N}$. Moreover, in terms of the Bernoulli numbers $B_{n}$ and the Euler polynomials $E_{n}(x)$ defined by the generating functions
(12) and

$$
\frac{2 e^{x z}}{e^{z}+1}=\sum_{n=0}^{\infty} E_{n}(x) \frac{z^{n}}{n !} \quad(|z|<\pi),
$$

respectively, it is known that (cf., e.g., [37, p. 29])

$$
E_{n}(0)=(-1)^{n} E_{n}(1)=\frac{2\left(1-2^{n+1}\right)}{n+1} B_{n+1},(n \in \mathbb{N}) .
$$

Thus, by combining (28) with the identity (11), we find that

$E_{2 n-1}(0)=\frac{4 \cdot(-1)^{n}}{(2 \pi)^{2 n}}(2 n-1) !\left(2^{2 n}-1\right) \zeta(2 n)$,

where $n \in \mathbb{N}$. If we apply the relationship (29), the series representation (26) can immediately be put in the following alternative form:

$$
\begin{aligned}
\zeta(2 n+1) & =(-1)^{n-1} \frac{2 \cdot(2 \pi)^{2 n}}{\left(2^{2 n}-1\right)\left(2^{2 n+1}-1\right)}\left[\frac{\log 2}{(2 n) !}\right. \\
& +\sum_{k=1}^{n-1} \frac{(-1)^{k}\left(2^{2 k}-1\right)}{(2 n-2 k) !} \frac{\zeta(2 k+1)}{\pi^{2 k}} \\
& \left.+\frac{1}{2} \sum_{k=1}^{\infty} \frac{(-1)^{k-1}}{(2 n+2 k) !}\left(\frac{\pi}{2}\right)^{2 k} E_{2 k-1}(0)\right]
\end{aligned}
$$

where $n \in \mathbb{N}$, which is a slightly modified and corrected version of a result proven, using a significantly different technique, by Tsumura [74, p. 383, Theorem B].

One other interesting combination of the series representations (22) and (24) leads us to the following variant of Tsumura's result (26) or (30):

$$
\begin{aligned}
\zeta(2 n+1) & =(-1)^{n-1} \frac{\pi^{2 n}}{2^{2 n+1}-1}\left[\frac{H_{2 n}-\log \left(\frac{1}{4} \pi\right)}{(2 n) !}\right. \\
& +\sum_{k=1}^{n-1} \frac{(-1)^{k}\left(2^{2 k+1}-1\right)}{(2 n-2 k) !} \frac{\zeta(2 k+1)}{\pi^{2 k}} \\
& \left.-4 \sum_{k=1}^{\infty} \frac{(2 k-1) !\left(2^{2 k-1}-1\right)}{(2 n+2 k) !} \frac{\zeta(2 k)}{2^{4 k}}\right],
\end{aligned}
$$

where $n \in \mathbb{N}$, which is essentially the same as the determinantal expression for $\zeta(2 n+1)$ derived 
by Ewell [20, p. 1010, Corollary 3] by employing an entirely different technique from ours.

A number of other similar combinations of the series representations (22) to (25) would yield some interesting companions of Ewell's result (31).

Next, by setting $t=1 / \mathrm{m}$ and differentiating both sides with respect to $s$, we find from the following obvious consequence of the series identity (14):

$$
\begin{aligned}
& \sum_{k=0}^{\infty} \frac{(s)_{2 k+1}}{(2 k+1) !} \zeta(s+2 k+1, a) t^{2 k+1} \\
& =\frac{1}{2}[\zeta(s, a-t)-\zeta(s, a+t)],(|t|<|a|)
\end{aligned}
$$

that

$$
\begin{aligned}
& \sum_{k=0}^{\infty} \frac{(s)_{2 k+1}}{(2 k+1) ! m^{2 k}}\left[\begin{array}{l}
\zeta^{\prime}(s+2 k+1, a) \\
+\zeta(s+2 k+1, a) \sum_{j=0}^{2 k} \frac{1}{s+j}
\end{array}\right] \\
& =\frac{m}{2} \frac{\partial}{\partial s}\left\{\zeta\left(s, a-\frac{1}{m}\right)-\zeta\left(s, a+\frac{1}{m}\right)\right\},
\end{aligned}
$$

where $\mathrm{m} \in \mathbb{N} \backslash\{1\}$. In the particular case when $m=2$, (33) immediately yields

$$
\begin{aligned}
& \sum_{k=0}^{\infty} \frac{(s)_{2 k+1}}{(2 k+1) ! 2^{2 k}}\left[\begin{array}{l}
\zeta^{\prime}(s+2 k+1, a) \\
+\zeta(s+2 k+1, a) \sum_{j=0}^{2 k} \frac{1}{s+j}
\end{array}\right] \\
& =-\left(a-\frac{1}{2}\right)^{-s} \log \left(a-\frac{1}{2}\right) .
\end{aligned}
$$

Upon letting $s \rightarrow-2 n-1(n \in \mathbb{N})$ in the further special of this last identity (34) when $a=1$, Wilton [57, p. 92] deduced the following series representation for $\zeta(2 n+1)$ (see also [27, p. 357 , Entry (54.6.9)]):

$$
\begin{aligned}
& \zeta(2 n+1)=(-1)^{n-1} \pi^{2 n}\left[\frac{H_{2 n+1}-\log \pi}{(2 n+1) !}\right. \\
& \left.+\sum_{k=1}^{n-1} \frac{(-1)^{k}}{(2 n-2 k+1) !} \frac{\zeta(2 k+1)}{\pi^{2 k}}\right],(n \in \mathbb{N}), \\
& \left.+2 \sum_{k=1}^{\infty} \frac{(2 k-1) !}{(2 n+2 k+1) !} \frac{\zeta(2 k)}{2^{2 k}}\right]
\end{aligned}
$$

which, in light of the elementary identity:

$$
\begin{aligned}
\frac{(2 k) !}{(2 n+2 k) !} & =\frac{(2 k-1) !}{(2 n+2 k-1) !} \\
& -2 n \frac{(2 k-1) !}{(2 n+2 k) !}, \quad(n \in \mathbb{N}),
\end{aligned}
$$

would combine with the result (22) to yield the following series representation:

$$
\begin{aligned}
& \zeta(2 n+1)=(-1)^{n} \frac{(2 \pi)^{2 n}}{n\left(2^{2 n+1}-1\right)} \\
& {\left[\begin{array}{l}
\sum_{k=1}^{n-1} \frac{(-1)^{k-1} k}{(2 n-2 k) !} \frac{\zeta(2 k+1)}{\pi^{2 k}} \\
+\sum_{k=0}^{\infty} \frac{(2 k) !}{(2 n+2 k) !} \frac{\zeta(2 k)}{2^{2 k}}
\end{array}\right],(n \in \mathbb{N}) .}
\end{aligned}
$$

This last series representation (37) is precisely the aforementioned main result of Cvijović and Klinowski [14, p. 1265, Theorem A]. As a matter of fact, in view of a known derivative formula [51, p. 389, Equation (21)], the series representation (37) is essentially the same as a result given earlier by Zhang and Williams [79, p. 1590, Equation (54)] (see also Zhang and Williams [79, p. 1591, Equation (57)] where an obviously more complicated (asymptotic) version of (37) was proven similarly).

In light of another elementary identity:

$$
\begin{aligned}
\frac{(2 k) !}{(2 n+2 k+1) !} & =\frac{(2 k-1) !}{(2 n+2 k) !} \\
& -(2 n+1) \frac{(2 k-1) !}{(2 n+2 k+1) !}
\end{aligned}
$$

where $n, k \in \mathbb{N}$, we can obtain the following yet another series representation for $\zeta(2 n+1)$ by applying (22) and (35):

$$
\begin{aligned}
& \zeta(2 n+1)=(-1)^{n} \frac{2 \cdot(2 \pi)^{2 n}}{(2 n-1) 2^{2 n}+1} \\
& {\left[\begin{array}{l}
\sum_{k=1}^{n-1} \frac{(-1)^{k-1} k}{(2 n-2 k+1) !} \frac{\zeta(2 k+1)}{\pi^{2 k}} \\
+\sum_{k=0}^{\infty} \frac{(2 k) !}{(2 n+2 k+1) !} \frac{\zeta(2 k)}{2^{2 k}}
\end{array}\right],(n \in \mathbb{N}),}
\end{aligned}
$$


which provides a significantly simpler (and much more rapidly convergent) version of the following other main result of Cvijović and Klinowski [14, p. 1265, Theorem B]:

$$
\zeta(2 n+1)=(-1)^{n} \frac{2 \cdot(2 \pi)^{2 n}}{(2 n) !} \sum_{k=0}^{\infty} \Omega_{n, k} \frac{\zeta(2 k)}{2^{2 k}},
$$

where $n \in \mathbb{N}$ and the coefficients $\Omega_{n, k}$ $\left(n \in \mathbb{N} ; k \in \mathbb{N}_{0}\right)$ are given explicitly as a finite sum of Bernoulli numbers [14, p. 1265, Theorem B(i)] (see, for details, Srivastava [51, pp. 393-394]):

$$
\Omega_{n, k}:=\sum_{j=0}^{2 n}\left(\begin{array}{c}
2 n \\
j
\end{array}\right) \frac{B_{2 n-j}}{(j+2 k+1)(j+1) \cdot 2^{j}},
$$

where $n \in \mathbb{N} ; k \in \mathbb{N}_{0}$.

\section{Other Families of Series Representations for$$
\zeta(\mathbf{2 n}+\mathbf{1})(\boldsymbol{n} \in \mathbb{N})
$$

In this section, we start once again from the identity (14) with (of course) $a=1, t= \pm 1 / m$, and $s$ replaced by $s+1$. Thus, by applying (15), we find yet another class of series identities including, for example,

$$
\sum_{k=1}^{\infty} \frac{(s+1)_{2 k}}{(2 k) !} \frac{\zeta(s+2 k)}{2^{2 k}}=\left(2^{s}-2\right) \zeta(s)
$$

and

$$
\begin{aligned}
& \sum_{k=1}^{\infty} \frac{(s+1)_{2 k}}{(2 k) !} \frac{\zeta(s+2 k)}{m^{2 k}} \\
& =\frac{1}{2 m}\left[\begin{array}{l}
m\left(m^{s}-3\right) \zeta(s)+\left(m^{s+1}-1\right) \zeta(s+1) \\
-2 \zeta\left(s+1, \frac{1}{m}\right) \\
-\sum_{j=2}^{m-2}\left\{m \zeta\left(s, \frac{j}{m}\right)+\zeta\left(s+1, \frac{j}{m}\right)\right\}
\end{array}\right]
\end{aligned}
$$

where $m \in \mathbb{N} \backslash\{1,2\}$. In fact, it is the series identity (42) which was first applied by Zhang and Williams [79] (and, subsequently, by Cvijović and Klinowski [14]) with a view to proving two (only seemingly different) versions of the series representation (37). Indeed, if we appeal to (43) with $m=4$, we can derive the following much more rapidly convergent series representation for $\zeta(2 n+1)$ (see [50, p. 9, Equation (41)]):

$$
\begin{aligned}
& \zeta(2 n+1)=(-1)^{n} \frac{2 \cdot(2 \pi)^{2 n}}{n\left(2^{4 n+1}+2^{2 n}-1\right)} \\
& {\left[\begin{array}{l}
\frac{4^{n-1}-1}{(2 n) !} B_{2 n} \log 2-\frac{2^{2 n-1}-1}{2(2 n-1) !} \zeta^{\prime}(1-2 n) \\
-\frac{4^{2 n-1}}{(2 n-1) !} \zeta^{\prime}\left(1-2 n, \frac{1}{4}\right) \\
+\sum_{k=1}^{n-1} \frac{(-1)^{k-1} k}{(2 n-2 k) !} \frac{\zeta(2 k+1)}{\left(\frac{1}{2} \pi\right)^{2 k}} \\
+\sum_{k=0}^{\infty} \frac{(2 k) !}{(2 n+2 k) !} \frac{\zeta(2 k)}{4^{2 k}}
\end{array}\right]}
\end{aligned}
$$

where $n \in \mathbb{N}$ and (and in what follows) a prime denotes the derivative of $\zeta(s)$ or $\zeta(s, a)$ with respect to $s$.

By virtue of the identities (36) and (38), the results (24) and (44) would lead us eventually to the following additional series representations for $\zeta(2 n+1)(n \in \mathbb{N})$ (see [50, p. 10, Equations (42) and (43)]):

$$
\begin{aligned}
& \zeta(2 n+1)=(-1)^{n-1}\left(\frac{\pi}{2}\right)^{2 n}\left[\frac{H_{2 n+1}-\log \left(\frac{1}{2} \pi\right)}{(2 n+1) !}\right. \\
& \left.+\frac{2\left(4^{n}-1\right)}{(2 n+2) !} B_{2 n+2} \log 2-\frac{2^{2 n+1}-1}{(2 n+1) !} \zeta^{\prime}(-2 n-1)\right] \\
& -\frac{2^{4 n+3}}{(2 n+1) !} \zeta^{\prime}\left(-2 n-1, \frac{1}{4}\right) \\
& +\sum_{k=1}^{n-1} \frac{(-1)^{k}}{(2 n-2 k+1) !} \frac{\zeta(2 k+1)}{\left(\frac{1}{2} \pi\right)^{2 k}} \\
& +2 \sum_{k=1}^{\infty} \frac{(2 k-1) !}{(2 n+2 k+1) !} \frac{\zeta(2 k)}{4^{2 k}}
\end{aligned}
$$


where $n \in \mathbb{N}$, and

$$
\begin{aligned}
& \zeta(2 n+1)=(-1)^{n} \frac{4 \cdot(2 \pi)^{2 n}}{n \cdot 4^{2 n+1}-2^{2 n}+1} \\
& \cdot\left[\begin{array}{l}
\frac{2^{2 n+1}-1}{2 \cdot(2 n) !} \zeta^{\prime}(-2 n-1) \\
+\frac{4^{2 n+1}}{(2 n) !} \zeta^{\prime}\left(-2 n-1, \frac{1}{4}\right) \\
-\frac{(2 n+1)\left(4^{n}-1\right)}{(2 n+2) !} B_{2 n+2} \log 2 \\
+\sum_{k=1}^{n-1} \frac{(-1)^{k-1} k}{(2 n-2 k+1) !} \frac{\zeta(2 k+1)}{\left(\frac{1}{2} \pi\right)^{2 k}} \\
+\sum_{k=0}^{\infty} \frac{(2 k) !}{(2 n+2 k+1) !} \frac{\zeta(2 k)}{4^{2 k}}
\end{array}\right],
\end{aligned}
$$

where $n \in \mathbb{N}$. Explicit expressions for the derivatives $\zeta^{\prime}(-2 n \pm 1)$ and $\zeta^{\prime}\left(-2 n \pm 1, \frac{1}{4}\right)$, occurring in the series representations (44), (45), and (46), can be found and substituted into these results in order to represent $\zeta(2 n+1)$ in terms of Bernoulli numbers and polynomials and various rapidly convergent series of the $\zeta$-functions (see, for details, the work by Srivastava [50, Section 3]).

Out of the four seemingly analogous results (24), (44), (45), and (46), the infinite series in (45) would obviously converge most rapidly, with its general term having the order estimate:

$$
O\left(k^{-2 n-2} \cdot 4^{-2 k}\right) \quad(k \rightarrow \infty ; n \in \mathbb{N}) .
$$

From the work by Srivastava and Tsumura [69], we recall the following three new members of the class of the series representations (24) and (45):

$$
\begin{aligned}
& \zeta(2 n+1)=(-1)^{n-1}\left(\frac{2 \pi}{3}\right)^{2 n} \\
& {\left[\begin{array}{l}
\frac{H_{2 n+1}-\log \left(\frac{2}{3} \pi\right)}{(2 n+1) !} \\
+\frac{(-1)^{n-1}}{\sqrt{3}(2 \pi)^{2 n+1} \zeta\left(2 \mathrm{n}+2, \frac{1}{3}\right)} \\
+\frac{\left(3^{2 n+2}-1\right) \pi}{2 \sqrt{3}(2 n+2) !} B_{2 n+2} \\
+\sum_{k=1}^{n-1} \frac{(-1)^{k}}{(2 n-2 k+1) !} \frac{\zeta(2 k+1)}{\left(\frac{2}{3} \pi\right)^{2 k}} \\
+2 \sum_{k=1}^{\infty} \frac{(2 k-1) !}{(2 n+2 k+1) !} \frac{\zeta(2 k)}{3^{2 k}}
\end{array}\right]}
\end{aligned}
$$


and [40]) obtained a number of remarkable integral expressions for $\zeta(3)$, including (for example) the following result [26, p. 1489 et seq.]:

$$
\zeta(3)=\frac{2 \pi^{2}}{7} \log 2-\frac{8}{7} \int_{0}^{\pi / 2} z^{2} \cot z \mathrm{~d} z .
$$

In fact, in view of the following series expansion [17, p. 51, Equation 1.20(3)]:

$$
z \cot z=-2 \sum_{k=0}^{\infty} \zeta(2 k)\left(\frac{z}{\pi}\right)^{2 k},(|z|<\pi),
$$

the result (51) equivalent to the series representation ( $c f$. the work by Dąbrowski [16, p. 202]; see also the paper by Chen and Srivastava [7, p. 191, Equation (60)]):

$$
\zeta(3)=\frac{2 \pi^{2}}{7}\left(\log 2+\sum_{k=0}^{\infty} \frac{\zeta(2 k)}{(k+1) \cdot 2^{2 k}}\right) .
$$

Moreover, if we integrate by parts, we easily find that

$$
\int_{0}^{\pi / 2} z^{2} \cot z \mathrm{~d} z=-2 \int_{0}^{\pi / 2} z \log \sin z \mathrm{~d} z
$$

so that the result (51) is equivalent also to the following integral representation:

$$
\zeta(3)=\frac{2 \pi^{2}}{7} \log 2+\frac{16}{7} \int_{0}^{\pi / 2} z \log \sin z \mathrm{~d} z,
$$

which was proven in the aforementioned 1772 paper by Euler (cf., e.g., [5, p. 1084]).

Furthermore, since

$$
i \cot i z=\operatorname{coth} z=\frac{2}{e^{2 z}-1}+1,(i:=\sqrt{-1}),
$$

by replacing $z$ in the known expansion (52) by $\frac{1}{2} i \pi z$, it is easily seen that (cf., e.g., [20, p. 25]; see also [17, p. 51, Equation 1.20(1)])

$\frac{\pi z}{e^{\pi z}-1}+\frac{\pi z}{2}=\sum_{k=0}^{\infty} \frac{(-1)^{k+1} \zeta(2 k)}{2^{2 k-1}} z^{2 k},(|z|<2)$.

Upon setting $z=i t$ in (57), multiplying both sides by $t^{m-1}(m \in \mathbb{N})$, and then integrating the resulting equation from $t=0$ to $t=\tau$ $(0<\tau<2)$, Srivastava [37] derived the following series representations for $\zeta(2 n+1)$ (see also the work by Srivastava et al. [62]):

$$
\begin{aligned}
& \zeta(2 n+1)=(-1)^{n-1} \frac{(2 \pi)^{2 n}}{(2 n) !\left(2^{2 n+1}-1\right)} \\
& \cdot\left[\begin{array}{l}
\log 2 \\
+\sum_{j=1}^{n-1}(-1)^{j}\left(\begin{array}{c}
2 n \\
2 j
\end{array}\right) \frac{(2 j) !\left(2^{2 j}-1\right)}{(2 \pi)^{2 j}} \zeta(2 j+1) \\
+\sum_{k=0}^{\infty} \frac{\zeta(2 k)}{(k+n) \cdot 2^{2 k}}
\end{array}\right],
\end{aligned}
$$

and

$$
\zeta(2 n+1)=(-1)^{n-1} \frac{(2 \pi)^{2 n}}{(2 n+1) !\left(2^{2 n}-1\right)}
$$

$$
\left[\begin{array}{c}
\log 2 \\
+\sum_{j=1}^{n-1}(-1)^{j}\left(\begin{array}{c}
2 n+1 \\
2 j
\end{array}\right) \\
\cdot \frac{(2 j) !\left(2^{2 j}-1\right)}{(2 \pi)^{2 j}} \zeta(2 j+1) \\
+\sum_{k=0}^{\infty} \frac{\zeta(2 k)}{\left(k+n+\frac{1}{2}\right) \cdot 2^{2 k}}
\end{array}\right],
$$

where $n \in \mathbb{N}$. Upon setting $n=1$, (59) immediately reduces to the following series representation for $\zeta(3)$ :

$$
\zeta(3)=\frac{2 \pi^{2}}{9}\left(\log 2+2 \sum_{k=0}^{\infty} \frac{\zeta(2 k)}{(2 k+3) \cdot 2^{2 k}}\right),
$$

which was proven independently by (among others) Glasser [23, p. 446, Equation (12)], Zhang and Williams [79, p. 1585, Equation (25)], and Dąbrowski [16, p. 206] (see also the work by Chen and Srivastava [7, p. 183, Equation (27)]). Furthermore, a special case of (58) when $n=1$ yields ( $c f$. Dąbrowski [16, p. 202]; see also Chen and Srivastava [7, 5, p. 191, Equation (60)])

$$
\zeta(3)=\frac{2 \pi^{2}}{7}\left(\log 2+\sum_{k=0}^{\infty} \frac{\zeta(2 k)}{(k+1) \cdot 2^{2 k}}\right) .
$$

In fact, in view of the following familiar sum:

$$
\sum_{k=0}^{\infty} \frac{\zeta(2 k)}{(2 k+1) \cdot 2^{2 k}}=-\frac{1}{2} \log 2,
$$


Euler's formula (9), that is,

$$
\zeta(3)=-\frac{4 \pi^{2}}{7} \sum_{k=0}^{\infty} \frac{\zeta(2 k)}{(2 k+1)(2 k+2) \cdot 2^{2 k}},
$$

is indeed a rather simple consequence of (61).

In passing, we find it worthwhile to remark that an integral representation for $\zeta(2 n+1)$, which is easily seen to be equivalent to the series representation (58), was given by Dąbrowski [16, p. 203, Equation (16)], who [16, p. 206] mentioned the existence of (but did not fully state) the series representation (59) as well. The series representation (58) was derived also in a paper by Borwein et al. (cf. [6, p. 269, Equation (57)]).

If we suitably combine the series occurring in (53), (60), and (62), it is not difficult to deduce several other series representations for $\zeta(3)$, which are analogous to Euler's formula (9), that is,

$$
\zeta(3)=-\frac{4 \pi^{2}}{7} \sum_{k=0}^{\infty} \frac{\zeta(2 k)}{(2 k+1)(2 k+2) \cdot 2^{2 k}} .
$$

More generally, since

$$
\begin{aligned}
& \frac{\lambda k^{2}+\mu k+\nu}{(2 k+2 n-1)(2 k+2 n)(2 k+2 n+1)} \\
& =\frac{\mathcal{A}}{2 k+2 n-1}+\frac{\mathcal{B}}{2 k+2 n}+\frac{\mathcal{C}}{2 k+2 n+1}
\end{aligned}
$$

where, for convenience,

$$
\begin{aligned}
\mathcal{A} & =\mathcal{A}_{n}(\lambda, \mu, \nu) \\
& :=\frac{1}{2}\left[\lambda n^{2}-(\lambda+\mu) n+\frac{1}{4}(\lambda+2 \mu+4 \nu)\right],
\end{aligned}
$$$$
\mathcal{B}=\mathcal{B}_{n}(\lambda, \mu, \nu):=-\left(\lambda n^{2}-\mu n+\nu\right),
$$

and

$$
\begin{aligned}
\mathcal{C} & =\mathcal{C}_{n}(\lambda, \mu, \nu) \\
& :=\frac{1}{2}\left[\lambda n^{2}+(\lambda-\mu) n+\frac{1}{4}(\lambda-2 \mu+4 \nu)\right],
\end{aligned}
$$

by applying (58), (59), and another result (proven by Srivastava [52, p. 341, Equation
(58)]):

$$
\begin{aligned}
& \sum_{j=1}^{n}(-1)^{j-1}\left(\begin{array}{c}
2 n+1 \\
2 j
\end{array}\right) \frac{(2 j) !\left(2^{2 j}-1\right)}{(2 \pi)^{2 j}} \zeta(2 j+1) \\
& =\log 2+\sum_{k=0}^{\infty} \frac{\zeta(2 k)}{\left(k+n+\frac{1}{2}\right) \cdot 2^{2 k}},\left(n \in \mathbb{N}_{0}\right),
\end{aligned}
$$

with $n$ replaced by $n-1$, Srivastava [52] derived the following unification of a large number of known (or new) series representations for $\zeta(2 n+1)(n \in \mathbb{N})$, including (for example) Euler's formula (9):

$$
\begin{aligned}
& \zeta(2 n+1) \\
& =\frac{(-1)^{n-1}(2 \pi)^{2 n}}{(2 n) !\left\{\left(2^{2 n+1}-1\right) \mathcal{B}+(2 n+1)\left(2^{2 n}-1\right) \mathcal{C}\right\}} \\
& \cdot\left[\begin{array}{l}
\frac{1}{4} \lambda \log 2+\sum_{j=1}^{n-1}(-1)^{j}\left(\begin{array}{l}
2 n-1 \\
2 j-2
\end{array}\right) \\
\cdot\left(\begin{array}{l}
2 j(2 j-1) \mathcal{A} \\
+[\lambda(4 n-1)-2 \mu] n j+\lambda n\left(n+\frac{1}{2}\right)
\end{array}\right) \\
\cdot \frac{(2 j-2) !\left(2^{2 j}-1\right)}{(2 \pi)^{2 j}} \zeta(2 j+1) \\
+\sum_{k=0}^{\infty} E(k)
\end{array}\right]
\end{aligned}
$$

where

$$
E(k):=\frac{\left(\lambda k^{2}+\mu k+\nu\right) \zeta(2 k)}{(2 k+2 n-1)(k+n)(2 k+2 n+1) \cdot 2^{2 k}}
$$

and $n \in \mathbb{N} ; \lambda, \mu, \nu \in \mathbb{C}$ and $\mathcal{A}, \mathcal{B}$, and $\mathcal{C}$ are given by (64), (65), and (66), respectively.

Numerous other interesting series representations for $\zeta(2 n+1)$, which are analogous to $(58)$ and (59), were also given by Srivastava et al. [62].

\section{Computationally Useful Deductions and Consequences}

In this section, we suitably specialize the parameter $\lambda, \mu$, and $\nu$ in (68) and then apply a 
rather elaborate scheme. We thus eventually arrive at the following remarkably rapidly convergent series representation for $\zeta(2 n+1)(n \in \mathbb{N})$, 349, Equation (3.50)]):

$$
\left[\begin{array}{c}
\zeta(2 n+1)=(-1)^{n-1} \frac{(2 \pi)^{2 n}}{(2 n) ! \Delta_{n}} \\
\sum_{j=1}^{n-1}(-1)^{j} \cdot \frac{(2 j) !\left(2^{2 j}-1\right)}{(2 \pi)^{2 j}} \zeta(2 j+1) \\
\cdot\left(\begin{array}{c}
\left\{(2 n-3) 2^{2 n+2}-2 n\right\} \\
\left(\begin{array}{c}
2 n-1 \\
2 j
\end{array}\right)-\left(\begin{array}{c}
2 n+2 \\
2 j
\end{array}\right) \\
\cdot\left\{\begin{array}{l}
2 n-1 \\
2 j-2
\end{array}\right) \\
-6 n\left(2^{2 n+3}-1\right) \\
\left(\begin{array}{l}
2 n \\
2 j
\end{array}\right)\left(\begin{array}{c}
2 n+3 \\
2 j
\end{array}\right) \\
\cdot\left\{\begin{array}{l}
2 n+1 \\
2 j-1
\end{array}\right)
\end{array}\right\} \\
+12 \sum_{k=0}^{\infty} \frac{\left(\xi_{n} k+\eta_{n}\right) \zeta(2 k)}{E(k)}
\end{array}\right\}
$$

where $n \in \mathbb{N}$,

$$
\begin{aligned}
E(k):= & (2 k+2 n-1)(2 k+2 n) \\
& \cdot(2 k+2 n+1)(2 k+2 n+2) \\
& \cdot(2 k+2 n+3) \cdot 2^{2 k}
\end{aligned}
$$

and, for convenience, which was derived by Srivastava [52, pp. 348-

In its special case when $n=1$, (69) yields the following (rather curious) series representation:

$$
\begin{aligned}
& \zeta(3)=-\frac{6 \pi^{2}}{23} \sum_{k=0}^{\infty} \\
& \frac{(98 k+121) \zeta(2 k)}{(2 k+1)(2 k+2)(2 k+3)(2 k+4)(2 k+5) \cdot 2^{2 k}}
\end{aligned}
$$

$$
\left[O\left(k^{-4} \cdot 2^{-2 k}\right) \quad(k \rightarrow \infty)\right]
$$

where the series obviously converges much more rapidly than that in each of the celebrated results (9) and (10), that is,

$$
\begin{gathered}
\zeta(3)=-\frac{4 \pi^{2}}{7} \sum_{k=0}^{\infty} \frac{\zeta(2 k)}{(2 k+1)(2 k+2) \cdot 2^{2 k}} \\
{\left[O\left(k^{-2} \cdot 2^{-2 k}\right) \quad(k \rightarrow \infty)\right]}
\end{gathered}
$$

and

$$
\begin{gathered}
\zeta(3)=\frac{5}{2} \sum_{k=1}^{\infty} \frac{(-1)^{k-1}}{k^{3}\left(\begin{array}{c}
2 k \\
k
\end{array}\right)} \\
{\left[O\left(k^{-\frac{5}{2}} \cdot 2^{-2 k}\right) \quad(k \rightarrow \infty)\right] .}
\end{gathered}
$$

An interesting companion of (73) in the following form:

$$
\begin{aligned}
& \zeta(3)=-\frac{120}{1573} \pi^{2} \sum_{k=0}^{\infty} \\
& \frac{8576 k^{2}+24286 k+17283}{E(k)} \frac{\zeta(2 k)}{2^{2 k}} \\
& \quad\left[O\left(k^{-5} \cdot 2^{-2 k}\right) \quad(k \rightarrow \infty)\right], \\
& E(k):=(2 k+1)(2 k+2) \\
& \cdot(2 k+3)(2 k+4) \\
& \cdot(2 k+5)(2 k+6)(2 k+7),
\end{aligned}
$$$$
\cdot\left\{\frac{1}{3}(2 n+1)\left(2 n^{2}-4 n+3\right)\left(2^{2 n}-1\right)-2^{2 n+1}+\frac{1}{w h}\right\} \text { here }
$$$$
-\left\{(2 n-3) 2^{2 n+2}-2 n\right\}
$$$$
\cdot\left\{2^{2 n+2}+n(2 n-3)\left(2^{2 n}-1\right)-1\right\} \text {, }
$$$$
\xi_{n}:=2\left\{(2 n-5) 2^{2 n+2}-2 n+1\right\},
$$

and

$$
\eta_{n}:=\left(4 n^{2}-4 n-7\right) 2^{2 n+2}-(2 n+1)^{2} .
$$

was deduced by Srivastava and Tsumura [71], who indeed presented an inductive construction of several general series representations for $\zeta(2 n+1)(n \in \mathbb{N})$ (see also [70]). 


\section{Numerical Verifications and Symbolic Computations \\ Based Upon Mathematica (Version 4.0)}

In this section, we first summarize the results of numerical verifications and symbolic computations with the series in (73) by using Mathematica (Version 4.0) for Linux:

$\operatorname{In}[1]:=(98 k+121) \operatorname{Zeta}[2 k] / E_{1}(k)$,

where

$$
E_{1}(k):=\left(\begin{array}{c}
(2 k+1)(2 k+2)(2 k+3) \\
\cdot(2 k+4)(2 k+5) 2\urcorner(2 k)
\end{array}\right)
$$

Out $[1]=\frac{(121+98 k) \text { Zeta }[2 k]}{E_{2}(k)}$,

where

$$
\begin{aligned}
E_{2}(k):= & 2^{2 k}(1+2 k)(2+2 k)(3+2 k) \\
& \cdot(4+2 k)(5+2 k) \\
\operatorname{In}[2]:= & \operatorname{Sum}[\%,\{k, 1, \text { Infinity }\}] / / \text { Simplify }
\end{aligned}
$$

Out $[2]=\frac{121}{240}-\frac{23 \mathrm{Zeta}[3]}{6 \mathrm{Pi}^{2}}$

$\operatorname{In}[3]:=\mathrm{N}[\%]$

Out $[3]=0.0372903$

$\operatorname{In}[4]:=\operatorname{Sum}[\mathrm{N}[\% 1] / /$ Evaluate, $\{k, 1,50\}]$

Out[4] $=0.0372903$

$\operatorname{In}[5]:=\mathrm{N}$ Sum $[\% 1 / /$ Evaluate, $\{k, 1$, Infinity $\}]$ In $[1]:=\operatorname{expr}$

Out $[5]=0.0372903$

Since

$$
\zeta(0)=-\frac{1}{2}
$$

Out[2] evidently validates the series representation (73) symbolically. Furthermore, our numerical computations in Out[3], Out[4], and Out[5], together, exhibit the fact that only 50 terms $(k=1$ to $k=50)$ of the series in (73) can produce an accuracy of as many as seven decimal places.

Our symbolic computations and numerical verifications with the series in (74) using Mathematica (Version 4.0) for Linux lead us to the following table:

\begin{tabular}{|c|c|}
\hline Number of Terms & Precision of Computation \\
\hline 4 & 6 \\
10 & 11 \\
20 & 18 \\
50 & 38 \\
98 & 69 \\
\hline
\end{tabular}

In fact, since the general term of the series in (74) has the following order estimate:

$$
O\left(2^{-2 k} \cdot k^{-5}\right) \quad(k \longrightarrow \infty),
$$

for getting $p$ exact digits, we must have

$$
2^{-2 k} \cdot k^{-5}<10^{-p} \text {. }
$$

Upon solving this inequality symbolically, we find that

$$
k \cong \frac{5}{\log 4} \operatorname{ProductLog}\left(\frac{10^{p / 5} \log 4}{5}\right),
$$

where the function ProductLog (also known as Lambert's function) is the solution of the equation:

$$
x e^{x}=a .
$$

Some relevant details about the symbolic computations and numerical verifications with the series in (74) using Mathematica (Version 4.0) for Linux are being summarized below.

$$
=(8576 k\urcorner 2+24286 k+17283) \operatorname{Zeta}[2 k] / E_{1}(k),
$$

where

$$
E_{1}(k):=\left(\begin{array}{c}
(2 k+1)(2 k+2)(2 k+3)(2 k+4) \\
\cdot(2 k+5)(2 k+6)(2 k+7) 2\urcorner(2 k)
\end{array}\right)
$$




$$
\begin{aligned}
& \text { Out [1] } \\
& =\left(17283+24286 k+8576 k^{2}\right) \operatorname{Zeta}[2 k] / E_{2}(k),
\end{aligned}
$$

where

$$
\begin{aligned}
E_{2}(k):= & 2^{2 k}(1+2 k)(2+2 k)(3+2 k) \\
& \cdot(4+2 k)(5+2 k)(6+2 k)(7+2 k)
\end{aligned}
$$

In [2] := Sum $[\operatorname{expr},\{k, 0$,infinity $\}] / /$ Simplify

$$
\text { Out }[2]=-\frac{1573}{120 \mathrm{Pi}^{2}} \text { Zeta[3] }
$$$$
\text { In }[3]:=\mathrm{N}\left[-1573 /\left(120 \mathrm{Pi}^{\urcorner} 2\right)\right. \text { Zeta[3], 50] }
$$$$
-\operatorname{Sum}[\operatorname{expr},\{k, 0,50\}]
$$

Out $[3]=4.00751120011 \cdot 10^{-38}$

$$
\begin{aligned}
\operatorname{In}[4]:=\mathrm{N}[ & -1573 /\left(120 \mathrm{Pi}^{\urcorner} 2\right) \text { Zeta[3], 100] } \\
& - \text { Sum }[\operatorname{expr},\{k, 0,50\}]
\end{aligned}
$$

Out $[4]=4.0075112001<$ skip $>3481 \cdot 10^{-38}$

Thus, clearly, the result does not change appreciably when we increase the precision of computation of the symbolic result from 50 to 100. This is expected, because of the following numerical computation of the last term for $k=50$ :

In [5] $:=\mathrm{N}[\operatorname{expr} / . k \rightarrow 50,50]$

Out [5] $=1.36085303749223768614438874545515$

$$
14233575702860179 \cdot 10^{-37}
$$

\section{The Hurwitz-Lerch Zeta Function $\boldsymbol{\Phi}(z, s, a)$ : Extensions and \\ Generalizations}

The potentially and computationally useful foregoing developments (which we have attempted to present here in a rather concise form) have essentially motivated a large number of further investigations on the subject, not only involving the Riemann Zeta function $\zeta(s)$ and the Hurwitz (or generalized) Zeta function $\zeta(s, a)$ (and their such relatives as the multiple Zeta functions and the multiple Gamma functions), but indeed also the substantially general HurwitzLerch Zeta function $\Phi(z, s, a)$ defined by ( $c f$., e.g., [17, p. 27. Eq. 1.11 (1)]; see also [57, p. 121, et seq.])

$$
\Phi(z, s, a):=\sum_{n=0}^{\infty} \frac{z^{n}}{(n+a)^{s}}
$$

$$
\begin{gathered}
\left(a \in \mathbb{C} \backslash \mathbb{Z}_{0}^{-} ; s \in \mathbb{C} \text { when }|z|<1 ;\right. \\
\Re(s)>1 \text { when }|z|=1) .
\end{gathered}
$$

Just as in the cases of the Riemann Zeta function $\zeta(s)$ and the Hurwitz (or generalized) Zeta function $\zeta(s, a)$, the Hurwitz-Lerch Zeta function $\Phi(z, s, a)$ can be continued meromorphically to the whole complex $s$-plane, except for a simple pole at $s=1$ with its residue 1 . It is also known that [17, p. 27, Equation 1.11 (3)]

$$
\begin{aligned}
\Phi(z, s, a) & =\frac{1}{\Gamma(s)} \int_{0}^{\infty} \frac{t^{s-1} \mathrm{e}^{-a t}}{1-z \mathrm{e}^{-t}} \mathrm{~d} t \\
& =\frac{1}{\Gamma(s)} \int_{0}^{\infty} \frac{t^{s-1} \mathrm{e}^{-(a-1) t}}{\mathrm{e}^{t}-z} \mathrm{~d} t
\end{aligned}
$$

$$
\begin{gathered}
(\Re(a)>0 ; \Re(s)>0 \text { when }|z| \leqq 1(z \neq 1) ; \\
\Re(s)>1 \text { when } z=1) .
\end{gathered}
$$

The Hurwitz-Lerch Zeta function $\Phi(z, s, a)$ defined by (75) contains, as its special cases, not only the Riemann Zeta function $\zeta(s)$ and the Hurwitz (or generalized) Zeta function $\zeta(s, a)$ [cf. Equations (1) and (2)]:

$$
\zeta(s)=\Phi(1, s, 1) \text { and } \zeta(s, a)=\Phi(1, s, a)
$$

and the Lerch Zeta function $\ell_{s}(\xi)$ defined by (see, for details, [17, Chapter I] and [57, Chapter 2])

$$
\ell_{s}(\xi):=\sum_{n=1}^{\infty} \frac{e^{2 n \pi i \xi}}{n^{s}}=e^{2 \pi i \xi} \Phi\left(e^{2 \pi i \xi}, s, 1\right)
$$

$(\xi \in \mathbb{R} ; \Re(s)>1)$, 
but also such other important functions of $A n$ alytic Function Theory as the Polylogarithmic function (or de Jonquière's function) $\operatorname{Li}_{s}(z)$ :

$$
\operatorname{Li}_{s}(z):=\sum_{n=1}^{\infty} \frac{z^{n}}{n^{s}}=z \Phi(z, s, 1)
$$

$$
(s \in \mathbb{C} \text { when }|z|<1 ; \Re(s)>1 \text { when }|z|=1)
$$

and the Lipschitz-Lerch Zeta function ( $c f$. [57, p. 122, Eq. 2.5 (11)]):

$$
\begin{aligned}
\phi(\xi, a, s): & =\sum_{n=0}^{\infty} \frac{e^{2 n \pi i \xi}}{(n+a)^{s}} \\
& =\Phi\left(e^{2 \pi i \xi}, s, a\right) \\
& =: L(\xi, s, a)
\end{aligned}
$$

$$
\begin{gathered}
\left(a \in \mathbb{C} \backslash \mathbb{Z}_{0}^{-} ; \Re(s)>0 \text { when } \xi \in \mathbb{R} \backslash \mathbb{Z} ;\right. \\
\Re(s)>1 \text { when } \xi \in \mathbb{Z}),
\end{gathered}
$$

which was first studied by Rudolf Lipschitz (1832-1903) and Matyáš Lerch (1860-1922) in connection with Dirichlet's famous theorem on primes in arithmetic progressions. For details, the interested reader should be referred, in connection with some of these developments, to the recent works including (among others) [2], [8] to [13], [22], [30], [31] and [36].

Yen et al. [78, p. 100, Theorem] derived the following sum-integral representation for the Hurwitz (or generalized) Zeta function $\zeta(s, a)$ defined by (2):

$$
\begin{gathered}
\zeta(s, a)=\frac{1}{\Gamma(s)} \sum_{j=0}^{k-1} \int_{0}^{\infty} \frac{t^{s-1} \mathrm{e}^{-(a+j) t}}{1-\mathrm{e}^{-k t}} \mathrm{~d} t \\
(k \in \mathbb{N} ; \Re(s)>1 ; \Re(a)>0),
\end{gathered}
$$

which, for $k=2$, was given earlier by Nishimoto et al. [41, p. 94, Theorem 4]. A straightforward generalization of the sum-integral representation (81) was given subsequently by Lin and Srivastava $[35$, p. 727, Eq. (7)] in the form:

$$
\Phi(z, s, a)=\frac{1}{\Gamma(s)} \sum_{j=0}^{k-1} z^{j} \int_{0}^{\infty} \frac{t^{s-1} \mathrm{e}^{-(a+j) t}}{1-z^{k} \mathrm{e}^{-k t}} \mathrm{~d} t
$$

$(k \in \mathbb{N} ; \Re(a)>0 ; \Re(s)>0$ when $|z| \leqq 1(z \neq 1) ;$ $\Re(s)>1$ when $z=1)$.

Motivated essentially by the sum-integral representations (81) and (82), a generalization of the Hurwitz-Lerch Zeta function $\Phi(z, s, a)$ was introduced and investigated by Lin and Srivastava [35] in the following form [35, p. 727, Eq. $(8)]$ :

$$
\Phi_{\mu, \nu}^{(\rho, \sigma)}(z, s, a):=\sum_{n=0}^{\infty} \frac{(\mu)_{\rho n}}{(\nu)_{\sigma n}} \frac{z^{n}}{(n+a)^{s}}
$$

$\left(\mu \in \mathbb{C} ; a, \nu \in \mathbb{C} \backslash \mathbb{Z}_{0}^{-} ; \rho, \sigma \in \mathbb{R}^{+} ; \rho<\sigma\right.$ when $s, z \in \mathbb{C} ; \rho=\sigma$ and $s \in \mathbb{C}$

when $|z|<\delta:=\rho^{-\rho} \sigma^{\sigma} ; \rho=\sigma$ and $\Re(s-\mu+\nu)>1$ when $|z|=\delta)$,

where $(\lambda)_{\nu}$ denotes the Pochhammer symbol defined in conjunction with (14) and (15). Clearly, we find from the definition (83) that

$$
\begin{aligned}
\Phi_{\nu, \nu}^{(\sigma, \sigma)}(z, s, a) & =\Phi_{\mu, \nu}^{(0,0)}(z, s, a) \\
& =\Phi(z, s, a)
\end{aligned}
$$

and

$$
\begin{aligned}
\Phi_{\mu, 1}^{(1,1)}(z, s, a) & =\Phi_{\mu}^{*}(z, s, a) \\
& :=\sum_{n=0}^{\infty} \frac{(\mu)_{n}}{n !} \frac{z^{n}}{(n+a)^{s}}
\end{aligned}
$$

$$
\begin{gathered}
\left(\mu \in \mathbb{C} ; a \in \mathbb{C} \backslash \mathbb{Z}_{0}^{-} ; s \in \mathbb{C} \text { when }|z|<1 ;\right. \\
\Re(s-\mu)>1 \text { when }|z|=1),
\end{gathered}
$$

where, as already noted by Lin and Srivastava [35], $\Phi_{\mu}^{*}(z, s, a)$ is a generalization of the Hurwitz-Lerch Zeta function considered by Goyal and Laddha [25, p. 100, Equation (4)]. For further results involving these classes of generalized Hurwitz-Lerch Zeta functions, see the recent works by Garg et al. [22] and Lin et al. [36].

A generalization of the above-defined Hurwitz-Lerch Zeta functions $\Phi(z, s, a)$ and $\Phi_{\mu}^{*}(z, s, a)$ was studied by Garg et al. [21] in the following form [21, p. 313, Eq. (10)]:

$$
\Phi_{\lambda, \mu ; \nu}(z, s, a):=\sum_{n=0}^{\infty} \frac{(\lambda)_{n}(\mu)_{n}}{(\nu)_{n} \cdot n !} \frac{z^{n}}{(n+a)^{s}}
$$


$\left(\lambda, \mu \in \mathbb{C} ; \nu, a \in \mathbb{C} \backslash \mathbb{Z}_{0}^{-} ; s \in \mathbb{C}\right.$ when $|z|<1 ;$ $\Re(s+\nu-\lambda-\mu)>1$ when $|z|=1)$.

By comparing the definitions (83) and (85), it is easily observed that the function $\Phi_{\lambda, \mu ; \nu}(z, s, a)$ studied by Garg et al. [21] does not provide a generalization of the function $\Phi_{\mu, \nu}^{(\rho, \sigma)}(z, s, a)$ which was introduced earlier by Lin and Srivastava [35]. Indeed, for $\lambda=1$, the function $\Phi_{\lambda, \mu ; \nu}(z, s, a)$ coincides with a special case of the function $\Phi_{\mu, \nu}^{(\rho, \sigma)}(z, s, a)$ when $\rho=\sigma=1$.

For the Riemann-Liouville fractional derivative operator $\mathcal{D}_{z}^{\mu}$ defined by (see, for example, [18, p. 181], [45] and [33, p. 70 et seq.])

$\mathcal{D}_{z}^{\mu}\{f(z)\}:=\left\{\begin{array}{l}\frac{1}{\Gamma(-\mu)} \int_{0}^{z}(z-t)^{-\mu-1} f(t) \mathrm{d} t \\ (\Re(\mu)<0) \\ \frac{d^{m}}{d z^{m}}\left\{\mathcal{D}_{z}^{\mu-m}\{f(z)\}\right\} \\ (m-1 \leqq \Re(\mu)<m(m \in \mathbb{N})),\end{array}\right.$

it is known that

$$
\mathcal{D}_{z}^{\mu}\left\{z^{\lambda}\right\}=\frac{\Gamma(\lambda+1)}{\Gamma(\lambda-\mu+1)} z^{\lambda-\mu}(\Re(\lambda)>-1),
$$

which, in view of the definition (83), yields the following fractional derivative formula for the generalized Hurwitz-Lerch Zeta function $\Phi_{\mu, \nu}^{(\rho, \sigma)}(z, s, a)$ with $\rho=\sigma$ [35, p. 730, Eq. (24)]:

$$
\begin{aligned}
\mathcal{D}_{z}^{\mu-\nu}\left\{z^{\mu-1} \Phi\left(z^{\sigma}, s, a\right)\right\} \\
=\frac{\Gamma(\mu)}{\Gamma(\nu)} z^{\nu-1} \Phi_{\mu, \nu}^{(\sigma, \sigma)}\left(z^{\sigma}, s, a\right) \\
\left(\Re(\mu)>0 ; \sigma \in \mathbb{R}^{+}\right) .
\end{aligned}
$$

In particular, when $\nu=\sigma=1$, the fractional derivative formula (89) would reduce at once to the following form:

$$
\Phi_{\mu}^{*}(z, s, a)=\frac{1}{\Gamma(\mu)} \mathcal{D}_{z}^{\mu-1}\left\{z^{\mu-1} \Phi(z, s, a)\right\},
$$

$$
(\Re(\mu)>0)
$$

which (as already remarked by Lin and Srivastava [35, p. 730]) exhibits the interesting (and useful) fact that $\Phi_{\mu}^{*}(z, s, a)$ is essentially a Riemann-Liouville fractional derivative of the classical Hurwitz-Lerch function
$\Phi(z, s, a)$. Moreover, it is easily deduced from the fractional derivative formula (88) that

$$
\begin{aligned}
\Phi_{\lambda, \mu ; \nu}(z, s, a) \\
=\frac{\Gamma(\nu)}{\Gamma(\lambda)} z^{1-\lambda} \mathcal{D}_{z}^{\lambda-\nu}\left\{z^{\lambda-1} \Phi_{\mu}^{*}(z, s, a)\right\} \\
=\frac{\Gamma(\nu)}{\Gamma(\lambda) \Gamma(\mu)} z^{1-\lambda} \\
\quad \cdot \mathcal{D}_{z}^{\lambda-\nu}\left\{z^{\lambda-1} \mathcal{D}_{z}^{\mu-1}\left\{z^{\mu-1} \Phi_{\mu}(z, s, a)\right\}\right\}
\end{aligned}
$$

which exhibits the hitherto unnoticed fact that the function $\Phi_{\lambda, \mu ; \nu}(z, s, a)$ studied by Garg et al. [21] is essentially a consequence of the classical Hurwitz-Lerch Zeta function $\Phi(z, s, a)$ when we apply the Riemann-Liouville fractional derivative operator $\mathcal{D}_{z}^{\mu}$ two times as indicated above (see also [67]). Many other explicit representations for $\Phi_{\mu}^{*}(z, s, a)$ and $\Phi_{\mu, \nu}^{(\rho, \sigma)}(z, s, a)$, including a potentially useful Eulerian integral representation of the first kind [35, p. 731, Eq. (28)], were proven by Lin and Srivastava [35].

A multiple (or, simply, $n$-dimentional) Hurwitz-Lerch Zeta function $\Phi_{n}(z, s, a)$ was studied recently by Choi et al. [9, p. 66, Eq. (6)]. Răducanu and Srivastava (see [43] and the references cited therein), on the other hand, made use of the Hurwitz-Lerch Zeta function $\Phi(z, s, a)$ in defining a certain linear convolution operator in their systematic investigation of various analytic function classes in Geometric Function Theory in Complex Analysis. Furthermore, Gupta et al. [26] revisited the study of the familiar Hurwitz-Lerch Zeta distribution by investigating its structural properties, reliability properties and statistical inference. These investigations by Gupta et al. [26] and others (see, for example, [53], [57], [60] and [61]), fruitfully using the Hurwitz-Lerch Zeta function $\Phi(z, s, a)$ and some of its above-mentioned generalizations, motivated Srivastava et al. [67] to present a further generalization and analogous investigation of a new family of Hurwitz-Lerch Zeta functions defined in the following form [67, p. 491, Equation (1.20)]:

$$
\Phi_{\lambda, \mu ; \nu}^{(\rho, \sigma, \kappa)}(z, s, a):=\sum_{n=0}^{\infty} \frac{(\lambda)_{\rho n}(\mu)_{\sigma n}}{(\nu)_{\kappa n} \cdot n !} \frac{z^{n}}{(n+a)^{s}}
$$


$\left(\lambda, \mu \in \mathbb{C} ; a, \nu \in \mathbb{C} \backslash \mathbb{Z}_{0}^{-} ; \rho, \sigma, \kappa \in \mathbb{R}^{+} ;\right.$

$\kappa-\rho-\sigma>-1$ when $s, z \in \mathbb{C}$;

$\kappa-\rho-\sigma=-1$ and $s \in \mathbb{C}$

when $|z|<\delta^{*}:=\rho^{-\rho} \sigma^{-\sigma} \kappa^{\kappa}$;

$\kappa-\rho-\sigma=-1$ and $\Re(s+\nu-\lambda-\mu)>1$

when $\left.|z|=\delta^{*}\right)$.

For the above-defined function in (92), Srivastava et al. [67] established various integral representations, relationships with the $\bar{H}$-function which is defined by means of a Mellin-Barnes type contour integral (see, for example, [65] and [67]), fractional derivative and analytic continuation formulas, as well as an extension of the generalized Hurwitz-Lerch Zeta function $\Phi_{\lambda, \mu ; \nu}^{(\rho, \sigma, \kappa)}(z, s, a)$ in (92). This natural further extension and generalization of the function $\Phi_{\lambda, \mu ; \nu}^{(\rho, \kappa)}(z, s, a)$ was indeed accomplished by introducing essentially arbirary numbers of numerator and denominator parameters in the definition (92). For this purpose, in addition to the symbol $\nabla^{*}$ defined by

$$
\nabla^{*}:=\left(\prod_{j=1}^{p} \rho_{j}^{-\rho_{j}}\right) \cdot\left(\prod_{j=1}^{q} \sigma_{j}^{\sigma_{j}}\right),
$$

the following notations will be employed:

$$
\Delta:=\sum_{j=1}^{q} \sigma_{j}-\sum_{j=1}^{p} \rho_{j}
$$

and

$$
\Xi:=s+\sum_{j=1}^{q} \mu_{j}-\sum_{j=1}^{p} \lambda_{j}+\frac{p-q}{2} .
$$

Then the extended Hurwitz-Lerch Zeta function

$$
\Phi_{\lambda_{1}, \cdots, \lambda_{p}, \mu_{1}, \cdots, \mu_{q}}^{\left(\rho_{1}, \cdots, \rho_{p}, \sigma_{1}, \cdots, \sigma_{q}\right)}(z, s, a)
$$

is defined by [67, p. 503, Equation (76)]

$$
\begin{aligned}
& \Phi_{\lambda_{1}, \cdots, \lambda_{p} ; \mu_{1}, \cdots, \mu_{q}}^{\left(\rho_{1}, \cdots, \rho_{p}, \sigma_{1}, \cdots, \sigma_{q}\right)}(z, s, a) \\
& :=\sum_{n=0}^{\infty} \frac{\prod_{j=1}^{p}\left(\lambda_{j}\right)_{n \rho_{j}}}{n ! \prod_{j=1}^{q}\left(\mu_{j}\right)_{n \sigma_{j}}} \frac{z^{n}}{(n+a)^{s}}
\end{aligned}
$$

$$
\begin{aligned}
& \left(p, q \in \mathbb{N}_{0} ; \lambda_{j} \in \mathbb{C}(j=1, \cdots, p) ;\right. \\
& a, \mu_{j} \in \mathbb{C} \backslash Z_{0}^{-}(j=1, \cdots, q) ; \\
& \rho_{j}, \sigma_{k} \in \mathbb{R}^{+}(j=1, \cdots, p ; k=1, \cdots, q) ; \\
& \Delta>-1 \text { when } s, z \in \mathbb{C} ; \\
& \Delta=-1 \text { and } s \in \mathbb{C} \text { when }|z|<\nabla^{*} ; \\
& \left.\Delta=-1 \text { and } \Re(\Xi)>\frac{1}{2} \text { when }|z|=\nabla^{*}\right) .
\end{aligned}
$$

The special case of the definition (95) when $p-1=q=1$ would obviously correspond to the above-investigated generalized HurwitzLerch Zeta function $\Phi_{\lambda, \mu ; \nu}^{(\rho, \sigma)}(z, s, a)$ defined by (92).

\section{If we set}

$p \mapsto p+1 \quad\left(\rho_{1}=\cdots=\rho_{p}=1 ; \quad \lambda_{p+1}=\rho_{p+1}=1\right)$

and

$$
q \mapsto q+1 \quad\left(\begin{array}{c}
\sigma_{1}=\cdots=\sigma_{q}=1 ; \\
\mu_{q+1}=\beta ; \quad \sigma_{q+1}=\alpha
\end{array}\right),
$$

then (95) reduces to the following generalized $M$-series which was recently introduced by Sharma and Jain [46] as follows:

$$
\begin{aligned}
& { }_{p}^{\alpha, \beta} M_{q}\left(a_{1}, \cdots, a_{p} ; b_{1}, \cdots, b_{q} ; z\right) \\
& =\sum_{k=0}^{\infty} \frac{\left(a_{1}\right)_{k} \cdots\left(a_{p}\right)_{k}}{\left(b_{1}\right)_{k} \cdots\left(b_{q}\right)_{k}} \frac{z^{k}}{\Gamma(\alpha k+\beta)} \\
& =\frac{1}{\Gamma(\beta)} \\
& \cdot p+1 \Psi_{q+1}^{*}\left[\begin{array}{c}
\left(a_{1}, 1\right), \cdots,\left(a_{p}, 1\right),(1,1) ; \\
\left(b_{1}, 1\right), \cdots,\left(b_{q}, 1\right),(\beta, \alpha) ;
\end{array}\right] .
\end{aligned}
$$

This last relationship (96) exhibits the fact that the so-called generalized $M$-series is, in fact, an obvious variant of the Fox-Wright function ${ }_{p} \Psi_{q}^{*}$ or ${ }_{p} \Psi_{q}^{*} \quad\left(p, q \in \mathbb{N}_{0}\right)$, which is a generalization of the familiar generalized hypergeometric function ${ }_{p} F_{q} \quad\left(p, q \in \mathbb{N}_{0}\right)$, with $p$ numerator parameters $a_{1}, \cdots, a_{p}$ and $q$ denominator parameters $b_{1}, \cdots, b_{q}$ such that

$$
\begin{aligned}
& a_{j} \in \mathbb{C}(j=1, \cdots, p), \\
& b_{j} \in \mathbb{C} \backslash \mathbb{Z}_{0}^{-}(j=1, \cdots, q),
\end{aligned}
$$


defined by (see, for details, [17, p. 183] and [64, p. 21]; see also [33, p. 56], [38, p. 30] and [63, p. 19])

$$
\begin{aligned}
& { }_{p} \Psi_{q}^{*}\left[\begin{array}{c}
\left(a_{1}, A_{1}\right), \cdots,\left(a_{p}, A_{p}\right) ; z \\
\left(b_{1}, B_{1}\right), \cdots,\left(b_{q}, B_{q}\right) ;
\end{array}\right] \\
& :=\sum_{n=0}^{\infty} \frac{\left(a_{1}\right)_{A_{1} n} \cdots\left(a_{p}\right)_{A_{p} n}}{\left(b_{1}\right)_{B_{1} n} \cdots\left(b_{q}\right)_{B_{q} n}} \frac{z^{n}}{n !} \\
& =\frac{\Gamma\left(b_{1}\right) \cdots \Gamma\left(b_{q}\right)}{\Gamma\left(a_{1}\right) \cdots \Gamma\left(a_{p}\right)} \\
& \cdot{ }_{p} \Psi_{q}\left[\begin{array}{c}
\left(a_{1}, A_{1}\right), \cdots,\left(a_{p}, A_{p}\right) ; \\
\left(b_{1}, B_{1}\right), \cdots,\left(b_{q}, B_{q}\right) ;
\end{array}\right]
\end{aligned}
$$

Each of the following results involving the extended Hurwitz-Lerch Zeta function

$$
\Phi_{\lambda_{1}, \cdots, \lambda_{p} ; \mu_{1}, \cdots, \mu_{q}}^{\left(\rho_{1}, \cdots, \rho_{p}, \sigma_{1}, \cdots, \sigma_{q}\right)}(z, s, a)
$$

can be proven by applying the definition (95) in precisely the same manner as for the corresponding result involving the general HurwitzLerch Zeta function $\Phi_{\lambda, \mu ; \nu}^{(\rho, \sigma, \kappa)}(z, s, a)$ (see, for details, [67, Section 6]).

$$
\begin{aligned}
& \Phi_{\lambda_{1}, \cdots, \lambda_{p} ; \mu_{1}, \cdots, \mu_{q}}^{\left(\rho_{1}, \cdots, \rho_{p}, \sigma_{1}, \cdots, \sigma_{q}\right)}(z, s, a)=\frac{1}{\Gamma(s)} \int_{0}^{\infty} t^{s-1} \mathrm{e}^{-a t} \\
& \left.\cdot{ }_{p} \Psi_{q}^{*}\left[\begin{array}{c}
\left(\lambda_{1}, \rho_{1}\right), \cdots,\left(\lambda_{p}, \rho_{p}\right) ; \\
\left(\mu_{1}, \sigma_{1}\right), \cdots,\left(\mu_{q}, \sigma_{q}\right) ;
\end{array}\right] \mathrm{e}^{-t}\right] \mathrm{d} t \quad(99)
\end{aligned}
$$

$$
\begin{gathered}
, q) ;, \quad(\min \{\Re(a), \Re(s)\}>0), \\
\Phi_{\lambda_{1}, \cdots, \lambda_{p} ; \mu_{1}, \cdots, \mu_{q}}^{\left(\rho_{1}, \cdots, \rho_{p}, \sigma_{1}, \cdots, \sigma_{q}\right)}(z, s, a)=\frac{\prod_{j=1}^{q} \Gamma\left(\mu_{j}\right)}{\prod_{j=1}^{p} \Gamma\left(\lambda_{j}\right)} \cdot \frac{1}{2 \pi i} \\
\cdot \int_{\mathfrak{L}} \frac{\Gamma(-\xi)\{\Gamma(\xi+a)\}^{s} \prod_{j=1}^{p} \Gamma\left(\lambda_{j}+\rho_{j} \xi\right)}{\{\Gamma(\xi+a+1)\}^{s} \prod_{j=1}^{q} \Gamma\left(\mu_{j}+\sigma_{j} \xi\right)}(-z)^{\xi} \mathrm{d} \xi
\end{gathered}
$$

In the particular case when

$$
A_{j}=B_{k}=1 \quad(j=1, \cdots, p ; k=1, \cdots, q),
$$

$$
(|\arg (-z)|<\pi)
$$

we have the following relationship (see, for details, [64, p. 21]):

$$
\begin{aligned}
& { }_{p} \Psi_{q}^{*}\left[\begin{array}{c}
\left(a_{1}, 1\right), \cdots,\left(a_{p}, 1\right) ; \\
\left(b_{1}, 1\right), \cdots,\left(b_{q}, 1\right) ;
\end{array}\right] \\
& ={ }_{p} F_{q}\left[\begin{array}{c}
a_{1}, \cdots, a_{p} ; \\
b_{1}, \cdots, b_{q} ;
\end{array}\right] \\
& =\frac{\Gamma\left(b_{1}\right) \cdots \Gamma\left(b_{q}\right)}{\Gamma\left(a_{1}\right) \cdots \Gamma\left(a_{p}\right)} \\
& \cdot{ }_{p} \Psi_{q}\left[\begin{array}{c}
\left(a_{1}, 1\right), \cdots,\left(a_{p}, 1\right) ; \\
\left(b_{1}, 1\right), \cdots,\left(b_{q}, 1\right) ;
\end{array}\right],
\end{aligned}
$$

in terms of the generalized hypergeometric function ${ }_{p} F_{q}\left(p, q \in \mathbb{N}_{0}\right)$. or, equivalently,

$$
\begin{aligned}
& \Phi_{\lambda_{1}, \cdots, \lambda_{p} ; \mu_{1}, \cdots, \mu_{q}}^{\left(\rho_{1}, \cdots, \rho_{q}, \sigma_{1}, \cdots, \sigma_{q}\right)}(z, s, a) \\
& =\frac{\prod_{j=1}^{q} \Gamma\left(\mu_{j}\right)}{\prod_{j=1}^{p} \Gamma\left(\lambda_{j}\right)} \cdot \bar{H}_{p+1, q+2}^{1, p+1}[z \mid E],
\end{aligned}
$$

where $E:=$

$$
\begin{gathered}
\left(1-\lambda_{1}, \rho_{1} ; 1\right), \cdots,\left(1-\lambda_{p}, \rho_{p} ; 1\right),(1-a, 1 ; s) \\
(0,1),\left(1-\mu_{1}, \sigma_{1} ; 1\right), \cdots,\left(1-\mu_{q}, \sigma_{q} ; 1\right),(-a, 1 ; s),
\end{gathered}
$$

provided that both sides of the assertions (99), (100) and (101) exist, the path of integration $\mathfrak{L}$ in (101) being a Mellin-Barnes type contour in the complex $\xi$-plane, which starts at the point $-i \infty$ and terminates at the point $i \infty$ with indentations, if necessary, in such a manner as 
to separate the poles of $\Gamma(-\xi)$ from the poles of $\Gamma\left(\lambda_{j}+\rho_{j} \xi\right) \quad(j=1, \cdots, p)$.

The $\bar{H}$-function representation given by (101) can be applied in order to derive various properties of the extended Hurwitz-Lerch Zeta function

$$
\Phi_{\lambda_{1}, \cdots, \lambda_{p}, \mu_{1}, \cdots, \mu_{q}}^{\left(\rho_{1}, \cdots, \rho_{p}, \sigma_{1}, \cdots, \sigma_{q}\right)}(z, s, a)
$$

from those of the $\bar{H}$-function. Thus, for example, by making use of the following fractionalcalculus result due to Srivastava et al. [65, p. 97, Eq. (17)]:

$$
\begin{gathered}
\mathcal{D}_{z}^{\nu}\left\{z^{\lambda-1} \bar{H}_{p, q}^{m, n}\left(\omega z^{\kappa}\right)\right\} \\
=z^{\lambda-\nu-1} \bar{H}_{p+1, q+1}^{m, n+1}\left[\omega z^{\kappa} \mid E\right], \\
(\Re(\lambda)>0 ; \kappa>0),
\end{gathered}
$$

where $E:=$

$$
\begin{gathered}
(1-\lambda, \kappa ; 1),\left(a_{j}, A_{j} ; \alpha_{j}\right)_{j=1}^{n},\left(a_{j}, A_{j}\right)_{j=n+1}^{p} \\
\left(b_{j}, B_{j}\right)_{j=1}^{m},\left(b_{j}, B_{j} ; \beta_{j}\right)_{j=m+1}^{q},(1-\lambda+\nu, \kappa ; 1),
\end{gathered}
$$

we readily obtain an extension of such fractional derivative formulas as (for example) (89) given by

$$
\begin{aligned}
& \mathcal{D}_{z}^{\nu-\tau}\left\{z^{\nu-1} \Phi_{\lambda_{1}, \cdots, \lambda_{p} ; \mu_{1}, \cdots, \mu_{q}}^{\left(\rho_{1}, \cdots, \rho_{p}, \sigma_{1}, \cdots, \sigma_{q}\right)}\left(z^{\kappa}, s, a\right)\right\} \\
&= \frac{\prod_{j=1}^{q} \Gamma\left(\mu_{j}\right)}{\prod_{j=1}^{p} \Gamma\left(\lambda_{j}\right)} z^{\tau-1} \cdot \bar{H}_{p+2, q+3}^{1, p+2}\left[-z^{\kappa} \mid E\right] \\
&= \frac{\Gamma(\nu)}{\Gamma(\tau)} z^{\tau-1} \Phi_{\lambda_{1}, \cdots, \lambda_{p}, \nu ; \mu_{1}, \cdots, \mu_{q}, \tau}^{\left(\rho_{1}, \cdots, \rho_{p}, \kappa, \sigma_{1}, \cdots, \sigma_{q}, \kappa\right)}\left(z^{\kappa}, s, a\right),
\end{aligned}
$$

where

$$
(\Re(\nu)>0 ; \kappa>0)
$$

$$
E:=\begin{gathered}
E_{1}, \cdots, E_{p},(1-\nu, \kappa ; 1),(1-a, 1 ; s) \\
(0,1), F_{1}, \cdots, F_{q},(1-\tau, \kappa ; 1),(-a, 1 ; s)
\end{gathered}
$$

with $E_{i}=\left(1-\lambda_{i}, \rho_{i} ; 1\right), F_{j}=\left(1-\mu_{j}, \sigma_{j} ; 1\right)(i=$ $\overline{1, p}, j=\overline{1, q})$. Finally, we present the following extension of a known result $[67$, p. 496 , Theorem 3] (see also [67, p. 505, Theorem 9].

Theorem. Let $\left(\alpha_{n}\right)_{n \in \mathbb{N}_{0}}$ be a positive sequence such that the following infinite series:

$$
\sum_{n=0}^{\infty} e^{-\alpha_{n} t}
$$

converges for any $t \in \mathbb{R}^{+}$. Then

$$
\begin{aligned}
\Phi_{\lambda_{1}, \cdots, \lambda_{p} ; \mu_{1}, \cdots, \mu_{q}}^{\left(\rho_{1}, \cdots, \rho_{p}, \sigma_{1}, \cdots, \sigma_{q}\right)}(z, s, a) \\
=\frac{1}{\Gamma(s)} \sum_{n=0}^{\infty} \int_{0}^{\infty} t^{s-1} e^{-\left(a-\alpha_{0}+\alpha_{n}\right) t} \\
\cdot\left(1-e^{-\left(\alpha_{n+1}-\alpha_{n}\right) t}\right) \\
\cdot{ }_{p} \Psi_{q}^{*}\left[\begin{array}{c}
\left(\lambda_{1}, \rho_{1}\right), \cdots,\left(\lambda_{p}, \rho_{p}\right) ; \\
\left(\mu_{1}, \sigma_{1}\right), \cdots,\left(\mu_{q}, \sigma_{q}\right) ;
\end{array}\right] \mathrm{d} t
\end{aligned}
$$

$$
(\min \{\Re(a), \Re(s)\}>0),
$$

provided that each member of (104) exists.

It would be nice and worthwhile to be able to extend the results presented in Sections 2 to 5 of this lecture to hold true for the HurwitzLerch Zeta function $\Phi(z, s, a)$ and for some of its generalizations given by the Lin-Srivastava Zeta function $\Phi_{\mu, \nu}^{(\rho, \sigma)}(z, s, a)$ and the extended Hurwitz-Lerch Zeta function

$$
\Phi_{\lambda_{1}, \cdots, \lambda_{p} ; \mu_{1}, \cdots, \mu_{q}}^{\left(\rho_{1}, \cdots, \rho_{p}, \sigma_{1}, \cdots, \sigma_{q}\right)}(z, s, a)
$$

defined by (95) for special values of the various parameters involved in the definitions (83) and (95). Several much more general open problems would involve the following $\lambda$-generalized Hurwitz-Lerch Zeta function whose investigation was initiated by Srivastava [56]:

$$
\begin{aligned}
& \Phi_{\lambda_{1}, \cdots, \lambda_{p} ; \mu_{1}, \cdots, \mu_{q}}^{\left(\rho_{1}, \cdots, \sigma_{1}\right)}(z, s, a ; b, \lambda) \\
& :=\frac{1}{\Gamma(s)} \int_{0}^{\infty} t^{s-1} \exp \left(-a t-\frac{b}{t^{\lambda}}\right) \\
& \left.\cdot{ }_{p} \Psi_{q}^{*}\left[\begin{array}{c}
\left(\lambda_{1}, \rho_{1}\right), \cdots,\left(\lambda_{p}, \rho_{p}\right) ; \\
\left(\mu_{1}, \sigma_{1}\right), \cdots,\left(\mu_{q}, \sigma_{q}\right) ;
\end{array}\right] e^{-t}\right] \mathrm{d} t, \\
& (\min \{\Re(a), \Re(s)\}>0 ; \Re(b) \geqq 0 ; \lambda \geqq 0),
\end{aligned}
$$

so that, obviously, we have the following relationship:

$$
\begin{aligned}
& \Phi_{\lambda_{1}, \cdots, \lambda_{p} ; \mu_{1}, \cdots, \mu_{q}}^{\left(\rho_{1}, \cdots, \rho_{p}, \sigma_{1}, \cdots, \sigma_{q}\right)}(z, s, a ; 0, \lambda) \\
& =\Phi_{\lambda_{1}, \cdots, \lambda_{p}, \sigma_{1}, \cdots, \mu_{q}}^{\left(\rho_{1}, \cdots, \rho_{p}, \cdots, s, a\right)} \\
& =e^{b} \Phi_{\lambda_{1}, \cdots, \lambda_{p} ; \mu_{1}, \cdots, \mu_{q}}^{\left(\rho_{1}, \cdots, \rho_{p}, \sigma_{1}, \cdots, \sigma_{q}\right)}(z, s, a ; b, 0) .
\end{aligned}
$$


Indeed, for the sake of the interested reader, we recall from Srivastava's work [56] the following explicit series representation formulas hold true for the $\lambda$-generalized Hurwitz-Lerch Zeta function

$$
\Phi_{\lambda_{1}, \cdots, \lambda_{p} ; \mu_{1}, \cdots, \mu_{q}}^{\left(\rho_{1}, \cdots, \rho_{q}, \sigma_{1}, \cdots, \sigma_{q}\right)}(z, s, a ; b, \lambda)
$$

defined by (105):

$$
\begin{aligned}
& \Phi_{\lambda_{1}, \cdots, \lambda_{p} ; \mu_{1}, \cdots, \mu_{q}}^{\left(\rho_{1}, \cdots, \rho_{p}, \sigma_{1}, \cdots, \sigma_{q}\right)}(z, s, a ; b, \lambda) \\
& =\frac{1}{\lambda \Gamma(s)} \sum_{n=0}^{\infty} \frac{\prod_{j=1}^{p}\left(\lambda_{j}\right)_{n \rho_{j}}}{(a+n)^{s} \cdot \prod_{j=1}^{q}\left(\mu_{j}\right)_{n \sigma_{j}}} \\
& \cdot H_{0,2}^{2,0}\left[(a+n) b^{\frac{1}{\lambda}} \mid \frac{z^{n}}{n !},\right.
\end{aligned}
$$

where $\lambda>0$ and $H_{p, q}^{m, n}[\cdot]$ denotes Fox's $H$ function (see, for details, [63].

The theory and applications of the various special as well as limit cases of the $\lambda$-generalized Hurwitz-Lerch Zeta function

$$
\Phi_{\lambda_{1}, \cdots, \lambda_{p} ; \mu_{1}, \cdots, \mu_{q}}^{\left(\rho_{1}, \cdots, \rho_{p}, \sigma_{1}, \cdots, \sigma_{q}\right)}(z, s, a ; b, \lambda),
$$

in addition to those mentioned above, can be found in (for example) the recent works [56] and [66], and indeed also in many of the earlier references which are cited in each of these recent works. Remarkably, just as its such aforementioned special cases as the Hurwitz-Lerch Zeta function $\Phi(z, s, a)$ and related Zeta functions, the $\lambda$-generalized Hurwitz-Lerch Zeta function

$$
\Phi_{\lambda_{1}, \cdots, \lambda_{p} ; \mu_{1}, \cdots, \mu_{q}}^{\left(\rho_{1}, \cdots, \rho_{p}, \sigma_{1}, \cdots, \sigma_{q}\right)}(z, s, a ; b, \lambda)
$$

defined by (105) is potentially useful and is currently being applied in many areas of the mathematical, statistical, physical and engineering sciences. The relevant details of such developments are easily accessible in the current literature on the subject.

\section{References}

[1] Abramowitz, M., \& Stegun, I. A. (1965). Handbook of mathematical functions: with formulas, graphs, and mathematical tables (Vol. 55). Courier Corporation.
[2] Alzer, H., Karayannakis, D., \& Srivastava, H. M. (2006). Series representations for some mathematical constants. Journal of Mathematical Analysis and Applications, 320(1), 145-162.

[3] Apéry, R. (1979). Irrationalité de $\zeta(2)$ et $\zeta(3)$, in Journées Arithmétiques de Luminy, $61,11-13$,

[4] Apostol, T. M. (1976). Introduction to Analytic Number Theory, Springer-Verlag, New York, Heidelberg and Berlin, 1976.

[5] Ayoub, R. (1974). Euler and the zeta function. The American Mathematical Monthly, 81(10), 1067-1086.

[6] Borwein, J. M., Bradley, D. M., \& Crandall, R. E. (2000). Computational strategies for the Riemann zeta function. Journal of Computational and Applied Mathematics, 121(1-2), 247-296.

[7] Chen, M. P., \& Srivastava, H. M. (1998). Some families of series representations for the Riemann $\zeta(3)$. Results in Mathematics, 33(3-4), 179-197.

[8] Choi, J., Cho, Y. J., \& Srivastava, H. M. (2004). Series involving the Zeta function and multiple Gamma functions. Applied Mathematics and Computation, 159(2), 509-537.

[9] Choi, J., Jang, D. S., \& Srivastava, H. M. (2008). A generalization of the HurwitzLerch Zeta function. Integral Transforms and Special Functions, 19(1), 65-79.

[10] Choi, J., \& Srivastava, H. M. (2005). Certain families of series associated with the Hurwitz-Lerch Zeta function. Applied Mathematics and Computation, 170(1), 399-409.

[11] Choi, J., \& Srivastava, H. M. (2005). Explicit evaluation of Euler and related sums. The Ramanujan Journal, 10(1), 51-70.

[12] Choi, J., \& Srivastava, H. M. (2014). Series involving the zeta functions and a family of generalized Goldbach-Euler series. The American Mathematical Monthly, 121(3), 229-236. 
[13] Choi, J., Srivastava, H. M., \& Adamchik, V. S. (2003). Multiple Gamma and related functions. Applied Mathematics and Computation, 134(2-3), 515-533.

[14] Cvijovic, D., \& Klinowski, J. (1997). New rapidly convergent series representations for $\zeta(2 n+1)$. Proceedings of the American Mathematical Society, 125(5), 1263-1271.

[15] Cvijovic, D., \& Srivastava, H. M. (2012). Limit Representations of Riemann's Zeta Function. The American Mathematical Monthly, 119(4), 324-330.

[16] Dabrowski, A. (1996). A note on values of the Riemann zeta function at positive odd integers. NIEUW ARCHIEF VOOR WISKUNDE, 14, 199-208.

[17] Erdélyi, A., Magnus, W., Oberhettinger, F., Tricomi, F. G. (1953). Higher Transcendental Functions. McGraw-Hill Book Company, New York, Toronto and London, Vol. I.

[18] Erdélyi, A., Magnus, W., Oberhettinger, F., Tricomi, F. G. (1954). Tables of Integral Transforms. McGraw-Hill Book Company, New York, Toronto and London, Vol. II.

[19] Ewell, J. A. (1990). A new series representation for $\zeta$ (3). Amer. Math. Monthly, 97, 219-220.

[20] Ewell, J. A. (1995). On the Zeta function values $\zeta(2 k+1), k=1,2, \cdots$. Rocky Mountain J. Math., 25, 1003-1012.

[21] Garg, M., Jain, K., \& Kalla, S. L. (2008). A further study of general Hurwitz-Lerch zeta function. Algebras Groups Geom, 25, 311-319.

[22] Garg, M., Jain, K., \& Srivastava, H. M. (2006). Some relationships between the generalized Apostol-Bernoulli polynomials and Hurwitz-Lerch Zeta functions. Integral Transforms and Special Functions, 17(11), 803-815.

[23] Glasser, M. L. (1968). Some integrals of the arctangent function. Mathematics of Computation, 22(102), 445-447.
[24] Gosper Jr, R. W. (1976). A calculus of series rearrangements, in Algorithms and Complexity: New Directions and Recent Results. Academic Press, New York, London and Toronto, 121-151.

[25] Goyal, S. P., Laddha, R. K. (1997). On the generalized Zeta function and the generalized Lambert function. Gaṇita Sandesh, 11, 99-108.

[26] Gupta, P. L., Gupta, R. C., Ong, S. H., \& Srivastava, H. M. (2008). A class of Hurwitz-Lerch Zeta distributions and their applications in reliability. Applied Mathematics and Computation, 196(2), 521-531.

[27] Hansen, E. R. (1975). A Table of Series and Products. Prentice-Hall, Englewood Cliffs, New Jersey.

[28] Hjortnaes, M. M. (1953). Overføring av rekken $\sum_{k=1}^{\infty}\left(1 / k^{3}\right)$ til et bestemt integral. Proceedings of the Twelfth Scandanavian Mathematical Congress, 211-213.

[29] Kanemitsu, S., Katsurada, M., Yoshimoto, M. (2000). On the Hurwitz-Lerch Zetafunction. Aequationes Mathematicae, 59, $1-19$.

[30] Kanemitsu, S., Kumagai, H., \& Yoshimoto, M. (2001). Sums involving the Hurwitz zeta function. The Ramanujan Journal, 5(1), 519.

[31] Kanemitsu, S., Kumagai, H., Srivastava, H. M., \& Yoshimoto, M. (2004). Some integral and asymptotic formulas associated with the Hurwitz Zeta function. Applied mathematics and computation, 154(3), 641-664.

[32] Katsurada, M. (1999). Rapidly convergent series representations for $\zeta(2 n+1)$ and their $\chi$-analogue. Acta Arithmetica, 90(1), 79-89.

[33] Kilbas, A. A. A., Srivastava, H. M., \& Trujillo, J. J. (2006). Theory and applications of fractional differential equations (Vol. 204). Elsevier Science Limited.

[34] Koblitz, N. (1977). p-Adic Numbers, $p$ Adic Analysis, and Zeta-Functions, Graduate Texts in Mathematics. Springer-Verlag, New York, Heidelberg and Berlin, 58. 
[35] Lin, S. D., \& Srivastava, H. M. (2004). Some families of the Hurwitz-Lerch Zeta functions and associated fractional derivative and other integral representations. Applied Mathematics and Computation, 154(3), 725-733.

[36] Lin, S. D., Srivastava, H. M., \& Wang, P. Y. (2006). Some expansion formulas for a class of generalized Hurwitz-Lerch zeta functions. Integral Transforms and Special Functions, 17(11), 817-827.

[37] Magnus, W., Oberhettinger, F., \& Soni, R. P. (2013). Formulas and theorems for the special functions of mathematical physics (Vol. 52). Springer Science \& Business Media.

[38] Mathai, A. M., Saxena, R. K., \& Haubold, H. J. (2009). The H-function: theory and applications. Springer Science \& Business Media.

[39] Nash, C., \& O'Connor, D. J. (1993). RaySinger torsion, topological field theories and the Riemann Zeta function at $s=3$. In Low-Dimensional Topology and Quantum Field Theory (pp. 279-288). Springer, Boston, MA.

[40] Nash, C., \& O'Connor, D. (1995). Determinants of Laplacians, the Ray-Singer torsion on lens spaces and the Riemann zeta function. Journal of Mathematical Physics, 36(3), 1462-1505.

[41] Nishimoto, K., Yen, C.-E, Lin, M.-L. (2002). Some integral forms for a generalized Zeta function. J. Fract. Calc. 22, 9197.

[42] Olver, F. W., Lozier, D. W., Boisvert, R. F., \& Clark, C. W. (Eds.). (2010). NIST handbook of mathematical functions hardback and CD-ROM. Cambridge university press.

[43] Răducanu, D., \& Srivastava, H. M. (2007). A new class of analytic functions defined by means of a convolution operator involving the Hurwitz-Lerch Zeta function. Integral Transforms and Special Functions, 18(12), 933-943.
[44] Ramaswami, V. (1934). Notes on Riemann's $\zeta$-function. J. London Math. Soc., 9, 165-169.

[45] Samko, S. G., Kilbas, A. A., \& Marichev, O. I. (1993). Fractional integrals and derivatives: theory and applications.

[46] Sharma, M., \& Jain, R. (2009). A note on a generalized M-series as a special function of fractional calculus. Fract. Calc. Appl. Anal, 12(4), 449-452.

[47] Srivastava, H. M. (1988). A unified presentation of certain classes of series of the Riemann Zeta function. Riv. Mat. Univ. Parma ( Ser. 4), 14, 1-23.

[48] Srivastava, H. M. (1988). Sums of certain series of the Riemann Zeta function. Journal of mathematical analysis and applications, 134(1), 129-140.

[49] Srivastava, H. M. (1997). Certain families of rapidly convergent series representations for $\zeta(2 n+1)$. Math. Sci. Res. Hot-Line, 1 (6), 1-6 (Research Announcement).

[50] Srivastava, H. M. (1998). Further series representations for $\zeta(2 n+1)$. Applied mathematics and computation, 97(1), 1-15.

[51] Srivastava, H. (1999). Some rapidly converging series for $\zeta(2 n+1)$. Proceedings of the American Mathematical Society, $127(2), 385-396$.

[52] Srivastava, H. M. (2000). Some simple algorithms for the evaluations and representations of the Riemann Zeta function at positive integer arguments. Journal of mathematical analysis and applications, 246(2), 331-351.

[53] Srivastava, H. M. (2000, July). Some formulas for the Bernoulli and Euler polynomials at rational arguments. In Mathematical Proceedings of the Cambridge Philosophical Society (Vol. 129, No. 1, pp. 77-84). Cambridge University Press.

[54] Srivastava, H. M. (2011). Some generalizations and basic (or $q-$ ) extensions of the Bernoulli, Euler and Genocchi polynomials. Appl. Math. Inform. Sci, 5(3), 390-444. 
[55] Hari, M. (2013). Generating relations and other results associated with some families of the extended Hurwitz-Lerch Zeta functions. SpringerPlus, 2(1), 67.

[56] Srivastava, H. M. (2014). A new family of the $\lambda$-generalized Hurwitz-Lerch zeta functions with applications. Appl. Math. Inf. Sci, 8(4), 1485-1500.

[57] Srivastava, H. M., \& Choi, J. (2001). Series associated with the zeta and related functions (Vol. 530). Springer Science \& Business Media.

[58] Srivastava, H. M., \& Choi, J. (2012). Zeta and $q$-Zeta functions and associated series and integrals. Elsevier.

[59] Srivastava, H. M., Gaboury, S., \& Tremblay, R. (2014). New relations involving an extended multiparameter Hurwitz-Lerch Zeta function with applications. International Journal of Analysis, 2014.

[60] Srivastava, H. M., Garg, M., \& Choudhary, S. (2010). A new generalization of the Bernoulli and related polynomials. Russian Journal of Mathematical Physics, 17(2), 251-261.

[61] Srivastava, H. M., Garg, M., \& Choudhary, S. (2011). Some new families of generalized Euler and Genocchi polynomials. Taiwanese Journal of Mathematics, 15(1), 283305 .

[62] Srivasata, H. M., Glasser, M. L., \& Adamchik, V. S. (2000). Some definite integrals associated with the Riemann zeta function. Z. Anal. Anwendungen, 19, 831-846.

[63] Srivastava, H. M., Gupta, K. C., Goyal, S. P. (1982). The $H$-Functions of One and Two Variables with Applications. South Asian Publishers, New Delhi and Madras.

[64] Srivastava, H. M., Karlsson, P. W. (1985). Multiple Gaussian Hypergeometric Series. Halsted Press (Ellis Horwood Limited, Chichester), John Wiley and Sons, New York, Chichester, Brisbane and Toronto.
[65] Srivastava, H. M., Lin, S. D., \& Wang, P. Y. (2006). Some fractional-calculus results for the $\bar{H}$-function associated with a class of Feynman integrals. Russian Journal of Mathematical Physics, 13(1), 94-100.

[66] Srivastava, H. M., Luo, M. J., \& Raina, R. K. (2013). New results involving a class of generalized Hurwitz-Lerch zeta functions and their applications. Turkish J. Anal. Number Theory, 1(1), 26-35.

[67] Srivastava, H. M., Saxena, R. K., Pogány, T. K., \& Saxena, R. (2011). Integral and computational representations of the extended Hurwitz-Lerch zeta function. Integral Transforms and Special Functions, 22(7), 487-506.

[68] Srivastava, H. M., Tomovski, Z., \& Leskovski, D. (2015). Some families of Mathieu-type series and Hurwitz-Lerch Zeta functions and associated probability distributions. Appl. Comput. Math, 14(3), 349-380.

[69] Srivastava, H. M., \& Tsumura, H. (2000). A certain class of rapidly convergent series representations for $\zeta(2 n+1)$. Journal of Computational and Applied Mathematics, 118(1-2), 323-335.

[70] Srivastava, H. M., Tsumura, H. (2000). New rapidly convergent series representations for $\zeta(2 n+1), L(2 n, \chi)$ and $L(2 n+1, \chi)$. Math. Sci. Res. Hot-Line, 4(7), 17-24 (Research Announcement).

[71] Srivastava, H. M., \& Tsumura, H. (2003). Inductive construction of rapidly convergent series representations for $\zeta(2 n+1)$. International journal of computer mathematics, 80(9), 1161-1173.

[72] Titchmarsh, E. C., Titchmarsh, E. C. T., \& Heath-Brown, D. R. (1986). The theory of the Riemann zeta-function. Oxford University Press.

[73] Tricomi, F. G. (1969). Sulla somma delle inverse delle terze e quinte potenze dei numeri naturali. Atti Accad. Naz. Lincei Rend. Cl. Sci. Fis. Mat. Natur. (Ser. 8), 47, 16-18. 
[74] Tsumura, H. (1994). On evaluation of the Dirichlet series at positive integers by $q$-calculation. Journal of Number Theory, 48(3), 383-391.

[75] Whittaker, E. T., Watson, G. N. (1927). A Course of Modern Analysis: An Introduction to the General Theory of Infinite Processes and of Analytic Functions; with an Account of the Principal Transcendental Functions. Fourth Edition, Cambridge University Press, Cambridge, London and New York.

[76] Wilton, J. R. (1922-1923). A proof of Burnside's formula for $\log \Gamma(x+1)$ and certain allied properties of Riemann's $\zeta$-function. Messenger Math., 52, 90-93.

[77] Witten, E. (1991). On quantum gauge theories in two dimensions. Communications in Mathematical Physics, 141(1), 153-209.

[78] Yen, C.-E, Lin, M.-L., Nishimoto, K. (2002). An integral form for a generalized Zeta function. J. Fract. Calc., 22, 99-102.

[79] Zhang, N.-Y., Williams, K. S. (1993). Some series representations of $\zeta(2 n+1)$. Rocky Mountain J. Math., 23, 1581-1592.

[80] Zhang, N.-Y., Williams, K. S. (1993). Some infinite series involving the Riemann Zeta function, in Analysis, Geometry and Groups: A Riemann Legacy Volume, Parts I and II (H. M. Srivastava and Th. M. Rassias, Editors), Part II, 691-712, Hadronic Press, Palm Harbor, Florida.

[81] Zhang, N.-Y., Williams, K. S. (1995). Values of the Riemann Zeta function and integrals involving $\log \left(2 \sinh \frac{\theta}{2}\right)$ and $\log \left(2 \sin \frac{\theta}{2}\right)$. Pacific J. Math., 168, 271-289. age of 19 years. He earned his Ph.D. degree in 1965 while he was a full-time member of the teaching faculty at J. N. V. University of Jodhpur (since 1963). Currently, Professor Srivastava holds the position of Professor Emeritus in the Department of Mathematics and Statistics at the University of Victoria in Canada, having joined the faculty there in 1969. Professor Srivastava has held numerous visiting positions at many universities and research institutes in different parts of the world. Having received several D.Sc. (honoris causa) degrees as well as honorary memberships and fellowships of many scientific academies around the world, he is also actively associated editorially with numerous international scientific research journals.

Professor Srivastava's research interests include several areas of pure and applied mathematical sciences such as (for example) Real and Complex Analysis, Fractional Calculus and Its Applications, Integral Equations and Transforms, Higher Transcendental Functions and Their Applications, $q$-Series and $q$-Polynomials, Analytic Number Theory, Analytic and Geometric Inequalities, Probability and Statistics, and Inventory Modelling and Optimization. He has published 24 books, monographs and edited volumes, 30 book (and encyclopedia) chapters, 48 papers in international conference proceedings, and over 1,200 scientific research journal articles.

Further details about Professor Srivastava's professional achievements and accomplishments, and honors, awards and distinctions, can be found at the following Web Site:

URL: http://www.math.uvic.ca/ harimsri/

\section{About Authors}

\section{Hari Mohan SRIVASTAVA (University of Victoria, Canada)}

Professor Hari Mohan Srivastava began his university-level teaching career right after having received his M.Sc. degree in 1959 at the
354 "This is an Open Access article distributed under the terms of the Creative Commons Attribution License, which permits unrestricted use, distribution, and reproduction in any medium provided the original work is 